\title{
Cationic Rh(I)/Modified-BINAP-Catalyzed Reactions of Carbonyl Compounds with 1,6-Diynes Leading to Dienones and Ortho-Functionalized Aryl Ketones
}

\author{
Ken Tanaka,* Yousuke Otake, Azusa Wada, Keiichi Noguchi, and Masao Hirano \\ Department of Applied Chemistry, Graduate School of Engineering, and Instrumentation \\ Analysis Center, Tokyo University of Agriculture and Technology, Koganei, Tokyo 184- \\ 8588, Japan
}

\section{General}

Anhydrous $\mathrm{CH}_{2} \mathrm{Cl}_{2}$ (No. 27099-7) and anhydrous $\left(\mathrm{CH}_{2} \mathrm{Cl}\right)_{2}$ (No. 28450-5) were obtained from Aldrich and used as received. $\mathrm{H}_{8}$-BINAP, Segphos, and DTBM-Segphos were obtained from Takasago International Corporation. All other reagents were obtained from commercial sources and used as received. All reactions were carried out under an atmosphere of argon or nitrogen in oven-dried glassware with magnetic stirring. Diynes $\mathbf{1 a},{ }^{1} \mathbf{1 b},{ }^{2} \mathbf{1 c},{ }^{3}$ and $\mathbf{1} \mathbf{d}^{4}$ were prepared according to the literatures. Carbonyl compounds $\mathbf{2} \mathbf{b},{ }^{5}$ $\mathbf{2 f},{ }^{6}$ and $\mathbf{2} \mathbf{h}^{7}$ were prepared according to the literatures.

\section{Rh-Catalyzed Reactions of 1,6-Diynes with Carbonyl Compounds (Table 1 and Scheme 1)}

General Procedure-1 (Table 1, entry 1). $\mathrm{H}_{8}$-BINAP $(9.5 \mathrm{mg}, 0.015 \mathrm{mmol})$ and $\left[\mathrm{Rh}(\mathrm{cod})_{2}\right] \mathrm{BF}_{4}(6.1 \mathrm{mg}, 0.015 \mathrm{mmol})$ were dissolved in $\mathrm{CH}_{2} \mathrm{Cl}_{2}(2.0 \mathrm{~mL})$, and the mixture was stirred at $\mathrm{rt}$ for $5 \mathrm{~min} . \mathrm{H}_{2}$ was introduced to the resulting solution in a Schlenk tube. After stirring at $\mathrm{rt}$ for $0.5 \mathrm{~h}$, the resulting solution was concentrated to dryness and dissolved in $\left(\mathrm{CH}_{2} \mathrm{Cl}\right)_{2}(0.5 \mathrm{~mL})$. To this solution was added dropwise a solution of $1 \mathrm{a}$ (70.9 $\mathrm{mg}, 0.300 \mathrm{mmol})$ and $2 \mathbf{a}(57.5 \mathrm{mg}, 0.330 \mathrm{mmol})$ in $\left(\mathrm{CH}_{2} \mathrm{Cl}\right)_{2}$ at $\mathrm{rt}$ and washed remaining substrates away by using $\left(\mathrm{CH}_{2} \mathrm{Cl}\right)_{2}(1.0 \mathrm{~mL})$. The mixture was stirred at $50{ }^{\circ} \mathrm{C}$ for $3 \mathrm{~h}$. The resulting solution was concentrated and purified by preparative TLC (hexane:ethyl acetate $=5: 1)$, which furnished 3aa (116.5 mg, $0.284 \mathrm{mmol}, 95 \%$ yield $)$ as a pale yellow oil.

3-Acetyl-4-(2,2-bisethoxycarbonyl-1-methylvinyl)cyclopent-3-ene-1,1-dicarboxylic acid dimethyl ester (3aa, Table 1, entry 1, 95\% yield)<smiles>CCOC(=O)C(C)=C(C)C1=C(C(C)=O)CC(C(C)=O)(C(OC)OC)C1</smiles> 
IR (neat) $2957,1732,1666,1247,735 \mathrm{~cm}^{-1} ;{ }^{1} \mathrm{H}$ NMR $\left(\mathrm{CDCl}_{3}, 300 \mathrm{MHz}\right) \delta 4.27$ (q, $J=$ $7.2 \mathrm{~Hz}, 2 \mathrm{H}), 4.12(\mathrm{q}, J=7.2 \mathrm{~Hz}, 2 \mathrm{H}), 3.75(\mathrm{~s}, 6 \mathrm{H}), 3.38-3.32(\mathrm{~m}, 4 \mathrm{H}), 2.21(\mathrm{~s}, 3 \mathrm{H}), 2.18(\mathrm{~s}$, $3 \mathrm{H}), 1.30(\mathrm{t}, J=7.2 \mathrm{~Hz}, 3 \mathrm{H}), 1.19(\mathrm{t}, J=7.2 \mathrm{~Hz}, 3 \mathrm{H}) ;{ }^{13} \mathrm{C} \mathrm{NMR}\left(\mathrm{CDCl}_{3}, 75 \mathrm{MHz}\right) \delta 195.1$, 171.1, 164.3, 163.8, 150.8, 149.6, 134.1, 125.9, 61.3, 61.2, 57.0, 53.0, 45.0, 41.0, 28.5, 19.7, 13.9, 13.8; HRMS (EI) calcd for $\mathrm{C}_{20} \mathrm{H}_{26} \mathrm{O}_{9}\left[\mathrm{M}-\mathrm{CO}_{2} \mathrm{Et}\right]^{+}$337.1288, found 337.1198.

3-Acetyl-4-(2-ethoxycarbonyl-1-methyl-2-phenylvinyl)cyclopent-3-ene-1,1dicarboxylic acid dimethylester (3ab, Table 1, entry 2, 98\% yield, $E: Z=1: 2$ )

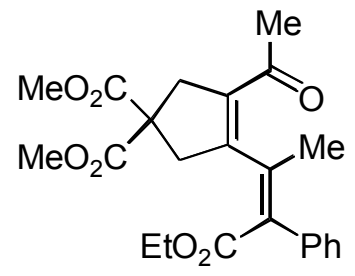

Pale yellow oil; IR (neat) 2954, 1738, 1660, 1268, $732 \mathrm{~cm}^{-1} ;{ }^{1} \mathrm{H}$ NMR $\left(\mathrm{CDCl}_{3}, 300\right.$ $\mathrm{MHz}) \delta 7.42-7.30(\mathrm{~m}, 3 \mathrm{H}), 7.29-7.23(\mathrm{~m}, 2 \mathrm{H}), 4.09(\mathrm{q}, J=7.2 \mathrm{~Hz}, 2 \mathrm{H}), 3.78(\mathrm{~s}, 6 \mathrm{H}), 3.47$ $(\mathrm{t}, J=1.5 \mathrm{~Hz}, 2 \mathrm{H}), 3.38(\mathrm{t}, J=1.5 \mathrm{~Hz}, 2 \mathrm{H}), 2.33(\mathrm{~s}, 3 \mathrm{H}), 1.87(\mathrm{~s}, 3 \mathrm{H}), 1.14(\mathrm{t}, J=7.2 \mathrm{~Hz}$, $3 \mathrm{H})$; $E$-isomer: ${ }^{1} \mathrm{H}$ NMR $\left(\mathrm{CDCl}_{3}, 300 \mathrm{MHz}\right) \delta 7.29-7.23(\mathrm{~m}, 3 \mathrm{H}), 7.16-7.10(\mathrm{~m}, 2 \mathrm{H}), 4.22$ $(\mathrm{q}, J=7.2 \mathrm{~Hz}, 2 \mathrm{H}), 3.65(\mathrm{~s}, 6 \mathrm{H}), 3.16(\mathrm{t}, J=1.5 \mathrm{~Hz}, 2 \mathrm{H}), 3.09(\mathrm{t}, J=1.5 \mathrm{~Hz}, 2 \mathrm{H}), 2.23(\mathrm{~s}$, $3 \mathrm{H}), 2.19(\mathrm{~s}, 3 \mathrm{H}), 1.25(\mathrm{t}, J=7.2 \mathrm{~Hz}, 3 \mathrm{H}) ;{ }^{13} \mathrm{C} \mathrm{NMR}\left(\mathrm{CDCl}_{3}, 75 \mathrm{MHz}\right) \delta 195.6,171.5$, $171.2,167.8,166.8,152.7,150.6,141.9,140.5,136.2,135.8,135.2,133.4,132.2,132.1$, 129.0, 128.29, 128.25, 128.0, 127.7, 127.6, 60.9, 57.0, 56.6, 53.0, 45.7, 45.1, 41.1, 28.7, 28.6, 20.2, 19.8, 14.1, 13.9; HRMS (EI) calcd for $\mathrm{C}_{23} \mathrm{H}_{26} \mathrm{O}_{7}[\mathrm{M}]^{+}$414.1679, found 414.1733.

3-Acetyl-4-(2-ethoxycarbonyl-1-methylpropenyl)cyclopent-3-ene-1,1-dicarboxylic acid dimethyl ester (3ac, Table 1, entry 3, 99\% yield, $E: Z=6: 1$ )

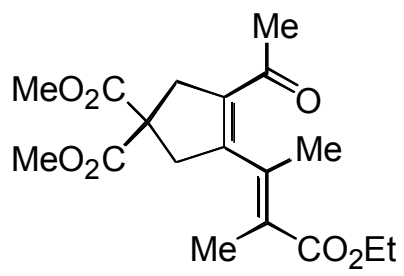

Pale yellow oil; IR (neat) 2955, 1737, 1660, 1250, $773 \mathrm{~cm}^{-1} ;{ }^{1} \mathrm{H}$ NMR $\left(\mathrm{CDCl}_{3}, 300\right.$ MHz) $\delta 4.02(\mathrm{q}, J=7.2 \mathrm{~Hz}, 2 \mathrm{H}), 3.69(\mathrm{~s}, 6 \mathrm{H}), 3.25(\mathrm{~s}, 4 \mathrm{H}), 2.07$ (s, 3H), 1.91 (s, 3H), 1.88 $(\mathrm{s}, 3 \mathrm{H}), 1.13(\mathrm{t}, J=7.2 \mathrm{~Hz}, 3 \mathrm{H}) ; Z$-isomer: ${ }^{1} \mathrm{H} \mathrm{NMR}\left(\mathrm{CDCl}_{3}, 300 \mathrm{MHz}\right) \delta 4.19(\mathrm{q}, J=7.2$ $\mathrm{Hz}, 2 \mathrm{H}), 3.69$ (s, 6H), 3.31-3.29 (m, 2H), 3.21-3.19 (m, 2H), 2.17 (s, 3H), 2.07 (s, 3H), $1.76-1.72(\mathrm{~m}, 3 \mathrm{H}), 1.28(\mathrm{t}, J=7.2 \mathrm{~Hz}, 3 \mathrm{H}) ;{ }^{13} \mathrm{C} \mathrm{NMR}\left(\mathrm{CDCl}_{3}, 75 \mathrm{MHz}\right) \delta 171.6,171.6$, 171.5, 167.4, 154.1, 141.0, 132.3, 126.0, 60.5, 56.8, 56.3, 53.0, 52.9, 45.5, 45.2, 41.1, 40.9, 28.3, 28.1, 19.3, 16.6, 15.1, 14.1, 14.0 ; HRMS (EI) calcd for $\mathrm{C}_{18} \mathrm{H}_{24} \mathrm{O}_{7}[\mathrm{M}]^{+} 352.1522$, found 352.1537 . 
dimethyl ester (3ad, Table 1, entry 4, 97\% yield, $E: Z=9: 1$ )

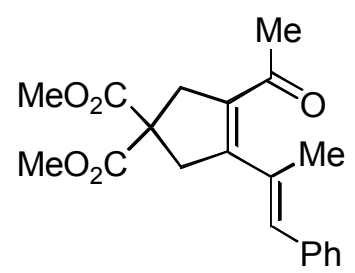

Pale yellow oil; IR (neat) 2954, 1737, 1686, 1267, $733 \mathrm{~cm}^{-1} ;{ }^{1} \mathrm{H}$ NMR $\left(\mathrm{CDCl}_{3}, 300\right.$ $\mathrm{MHz}) \delta 7.40-7.16(\mathrm{~m}, 5 \mathrm{H}), 6.44(\mathrm{~s}, 1 \mathrm{H}), 3.77(\mathrm{~s}, 6 \mathrm{H}), 3.40(\mathrm{~s}, 4 \mathrm{H}), 2.29(\mathrm{~s}, 3 \mathrm{H}), 2.07(\mathrm{~s}$, $3 \mathrm{H})$; Z-isomer: ${ }^{1} \mathrm{H}$ NMR $\left(\mathrm{CDCl}_{3}, 300 \mathrm{MHz}\right) \delta 7.40-7.16(\mathrm{~m}, 5 \mathrm{H}), 6.41(\mathrm{~s}, 1 \mathrm{H}), 3.72(\mathrm{~s}, 6 \mathrm{H})$, $3.37(\mathrm{t}, J=1.8 \mathrm{~Hz}, 2 \mathrm{H}), 3.30(\mathrm{t}, J=1.8 \mathrm{~Hz}, 2 \mathrm{H}), 2.22(\mathrm{~s}, 3 \mathrm{H}), 2.07(\mathrm{~s}, 3 \mathrm{H}) ;{ }^{13} \mathrm{C} \mathrm{NMR}$ $\left(\mathrm{CDCl}_{3}, 75 \mathrm{MHz}\right) \delta 197.8,196.0,171.6,171.5,153.0,151.6,136.4,136.2,135.4,134.7$, $133.1,133.0,130.5,128.8,128.4,128.2$, 127.6, 127.1, 127.0, 56.3, 56.0, 52.9, 52.8, 45.5, 44.9, 41.6, 41.2, 29.1, 28.0, 24.5, 16.9; HRMS (EI) calcd for $\mathrm{C}_{20} \mathrm{H}_{22} \mathrm{O}_{5}[\mathrm{M}]^{+} 342.1467$, found 342.1513 .

3-Acetyl-4-(1-methylbuta-1,3-dienyl)cyclopent-3-ene-1,1-dicarboxylic acid diethyl ester (3ae, Table 1, entry 5, 55\% yield, $E: Z=>20: 1$ )<smiles>C=C/C=C(/C)C1=C(C(C)=O)CC(C(=O)OCC)(C(=O)OCC)C1</smiles>

Yellow oil; IR (neat) 2954, 1735, 1660, 1266, $736 \mathrm{~cm}^{-1} ;{ }^{1} \mathrm{H}$ NMR $\left(\mathrm{CDCl}_{3}, 300 \mathrm{MHz}\right) \delta$ 6.64-6.52 (m, 1H), 6.00-5.97 (m, 1H), 5.28-5.20 (m, 2H), $3.73(\mathrm{~s}, 6 \mathrm{H}), 3.32$ (t, J = 1.8 Hz, $2 \mathrm{H}), 3.28(\mathrm{t}, J=1.8 \mathrm{~Hz}, 2 \mathrm{H}), 2.18(\mathrm{~s}, 3 \mathrm{H}), 1.91(\mathrm{~d}, J=1.2 \mathrm{~Hz}, 3 \mathrm{H}) ;{ }^{13} \mathrm{C} \mathrm{NMR}\left(\mathrm{CDCl}_{3}, 75\right.$ $\mathrm{MHz}) \delta 198.2,171.7,151.9,134.7,132.6,132.0,131.4,119.6,56.4,53.0,45.3,41.7,29.2$, 29.1, 15.7; HRMS (EI) calcd for $\mathrm{C}_{16} \mathrm{H}_{20} \mathrm{O}_{5}[\mathrm{M}]^{+}$292.1311, found 292.1280.

3-Acetyl-4-(1,2-dimethylpropenyl)cyclopent-3-ene-1,1-dicarboxylic acid dimethyl ester (3af) and 1,3,3,4-tetramethyl-3,5-dihydro-7H-cyclopenta[c]pyran-6,6dicarboxylic acid dimethyl ester (3af, Table 1, entry 6, 51\% yield, dienone:ether = 3:1)<smiles>CC(=O)C1=C(C(C)=O)CC(C(C)=O)(C(C)=O)C1</smiles><smiles>COC(=O)C1(C(C)(C)C)CC2=C(C)OC(C)(C)C(C)=C2C1</smiles>

Pale yellow oil; IR (neat) 2954, 1732, 1659, 1619, $733 \mathrm{~cm}^{-1} ;{ }^{1} \mathrm{H}$ NMR $\left(\mathrm{CDCl}_{3}, 300\right.$ $\mathrm{MHz}) \delta 3.72(\mathrm{~s}, 6 \mathrm{H}), 3.30(\mathrm{~s}, 2 \mathrm{H}), 3.18(\mathrm{~s}, 2 \mathrm{H}), 2.16(\mathrm{~d}, J=0.3 \mathrm{~Hz}, 3 \mathrm{H}), 1.77(\mathrm{~s}, 3 \mathrm{H}), 1.70$ (s, 3H), 1.57 (s, 3H); ether-isomer: ${ }^{1} \mathrm{H}$ NMR $\left(\mathrm{CDCl}_{3}, 300 \mathrm{MHz}\right) \delta 3.71(\mathrm{~s}, 6 \mathrm{H}), 2.94(\mathrm{~s}, 2 \mathrm{H})$, $2.90(\mathrm{~s}, 2 \mathrm{H}), 1.73(\mathrm{~s}, 3 \mathrm{H}), 1.59(\mathrm{~s}, 3 \mathrm{H}), 1.24(\mathrm{~s}, 6 \mathrm{H}) ;{ }^{13} \mathrm{C} \mathrm{NMR}\left(\mathrm{CDCl}_{3}, 75 \mathrm{MHz}\right) \delta 196.8$, 
$171.9,155.0,141.7,134.4,129.3,128.8,124.4,117.3,78.9,59.6,56.0,52.9,52.7,45.9$, $40.8,39.0,36.9,35.6,34.04,34.01,27.6,24.7,21.4,19.7,17.3,17.0,14.2$; HRMS (EI) calcd for $\mathrm{C}_{16} \mathrm{H}_{22} \mathrm{O}_{5}[\mathrm{M}]^{+}$294.1467, found 294.1415.

\section{2-[1-\{4-Acetyl-1-(toluene-4-sulfonyl)-2,5-dihydro-1H-pyrrol-3-} yl\}ethylidene]malonic acid diethyl ester (3ba, Table 1, entry $8,88 \%$ yield)<smiles>CCOC(=O)C(OCC)=C(C)C1=C(C(C)=O)CN([AlH2])C1</smiles>

Pale yellow oil; IR (neat) 2983, 1726, 1668, 1350, $1166 \mathrm{~cm}^{-1} ;{ }^{1} \mathrm{H}$ NMR $\left(\mathrm{CDCl}_{3}, 300\right.$ MHz) $\delta 7.74(\mathrm{~d}, J=8.4 \mathrm{~Hz}, 2 \mathrm{H}), 7.36(\mathrm{~d}, J=8.4 \mathrm{~Hz}, 2 \mathrm{H}), 4.43-4.39(\mathrm{~m}, 2 \mathrm{H}), 4.38-4.33(\mathrm{~m}$, $2 \mathrm{H}), 4.28$ (q, $J=7.2 \mathrm{~Hz}, 2 \mathrm{H}), 3.48(\mathrm{q}, J=7.2 \mathrm{~Hz}, 2 \mathrm{H}), 2.44(\mathrm{~s}, 3 \mathrm{H}), 2.18(\mathrm{~s}, 3 \mathrm{H}), 2.11$ (s, $3 \mathrm{H}), 1.31(\mathrm{t}, J=7.2 \mathrm{~Hz}, 3 \mathrm{H}), 1.08(\mathrm{t}, J=7.2 \mathrm{~Hz}, 3 \mathrm{H}) ;{ }^{13} \mathrm{C} \mathrm{NMR}\left(\mathrm{CDCl}_{3}, 75 \mathrm{MHz}\right) \delta 193.3$, 163.9, 163.2, 146.6, 146.3, 143.8, 133.4, 132.1, 129.8, 127.7, 127.5, 61.6, 61.4, 58.2, 55.3, 28.4, 21.4, 19.9, 13.9, 13.6; HRMS (EI) calcd for $\mathrm{C}_{22} \mathrm{H}_{27} \mathrm{NO}_{7} \mathrm{~S}[\mathrm{M}]^{+} 449.1508$, found 449.1466 .

2-[1-(4-Propionyl-2,5-dihydrofuran-3-yl)propylidene]malonic acid diethyl ester (3ca, Table 1, entry 9, $84 \%$ yield $)^{8}$<smiles>CCOC(=O)C(CC)=C(C(=O)OCC)C1=C(C(=O)CC)COC1</smiles>

Pale yellow oil; ${ }^{1} \mathrm{H}$ NMR $\left(\mathrm{CDCl}_{3}, 300 \mathrm{MHz}\right) \delta 4.93(\mathrm{t}, J=4.5 \mathrm{~Hz}, 2 \mathrm{H}), 4.84(\mathrm{t}, J=4.5$ $\mathrm{Hz}, 2 \mathrm{H}), 4.28(\mathrm{q}, J=7.2 \mathrm{~Hz}, 2 \mathrm{H}), 4.14(\mathrm{q}, J=7.2 \mathrm{~Hz}, 2 \mathrm{H}), 2.56(\mathrm{q}, J=7.2 \mathrm{~Hz}, 2 \mathrm{H}), 2.50(\mathrm{q}$, $J=7.2 \mathrm{~Hz}, 2 \mathrm{H}), 1.29(\mathrm{t}, J=7.2 \mathrm{~Hz}, 3 \mathrm{H}), 1.21(\mathrm{t}, J=7.2 \mathrm{~Hz}, 3 \mathrm{H}), 1.11(\mathrm{t}, J=7.2 \mathrm{~Hz}, 3 \mathrm{H})$, $1.03(\mathrm{t}, J=7.2 \mathrm{~Hz}, 3 \mathrm{H}) ;{ }^{13} \mathrm{C} \mathrm{NMR}\left(\mathrm{CDCl}_{3}, 75 \mathrm{MHz}\right) \delta 196.7,164.4,163.7,152.5,146.6$, $134.2,126.7,79.5,76.7,61.51,61.46,34.7,27.9,14.0,13.9,12.3,7.4$.

3-Acetyl-4-(1-methyl-4-phenylbut-1-en-3-ynyl)cyclopent-3-ene-1,1-dicarboxylic acid dimethyl ester (3ah, Scheme 1, 90\% yield, $E: Z=>20: 1$ )

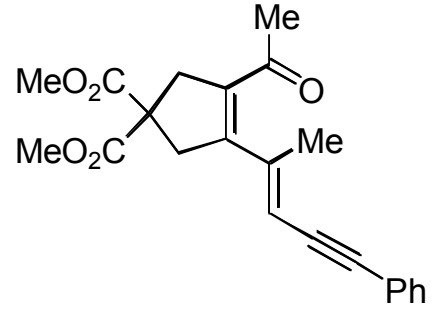


Pale yellow oil; IR (neat) 2954, 1736, 1660, 1265, $759 \mathrm{~cm}^{-1} ;{ }^{1} \mathrm{H}$ NMR $\left(\mathrm{CDCl}_{3}, 300\right.$ $\mathrm{MHz}) \delta 7.48-7.42(\mathrm{~m}, 2 \mathrm{H}), 7.36-7.30(\mathrm{~m}, 3 \mathrm{H}), 5.73-5.71(\mathrm{~m}, 1 \mathrm{H}), 3.76(\mathrm{~s}, 6 \mathrm{H}), 3.37(\mathrm{t}, J=$ $1.8 \mathrm{~Hz}, 2 \mathrm{H}), 3.33(\mathrm{t}, J=1.8 \mathrm{~Hz}, 2 \mathrm{H}), 2.28(\mathrm{~s}, 3 \mathrm{H}), 2.14(\mathrm{~d}, J=1.2 \mathrm{~Hz}, 3 \mathrm{H}) ;{ }^{13} \mathrm{C} \mathrm{NMR}$ $\left(\mathrm{CDCl}_{3}, 75 \mathrm{MHz}\right) \delta 198.1,171.4,148.6,144.1,135.7,131.3,128.3,128.2,123.0,111.3$, 96.5, 86.2, 56.4, 53.0, 45.0, 42.0, 29.3, 18.5; HRMS (EI) calcd for $\mathrm{C}_{21} \mathrm{H}_{19} \mathrm{O}_{4}[\mathrm{M}-\mathrm{OMe}]^{+}$ 335.1283 , found 335.1332 .

5-Acetyl-4,7-dimethyl-6-phenylindan-2,2-dicarboxylic acid dimethyl ester (4, Scheme 1, 99\% yield)

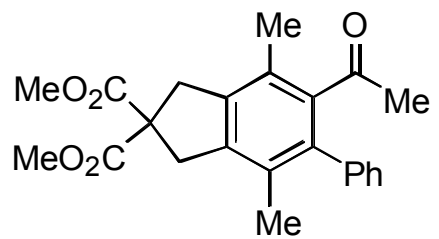

Colorless solid; Mp 160.0-161.2 ${ }^{\circ} \mathrm{C}$; IR (neat) 2953, 1737, 1694, 1248, $733 \mathrm{~cm}^{-1} ;{ }^{1} \mathrm{H}$ NMR $\left(\mathrm{CDCl}_{3}, 300 \mathrm{MHz}\right) \delta 7.40-7.30(\mathrm{~m}, 3 \mathrm{H}), 7.19-7.14(\mathrm{~m}, 2 \mathrm{H}), 3.79(\mathrm{~s}, 6 \mathrm{H}), 3.61(\mathrm{~s}$, 4H), 2.15 (s, 3H), $2.00(\mathrm{~s}, 3 \mathrm{H}), 1.84(\mathrm{~s}, 3 \mathrm{H}) ;{ }^{13} \mathrm{C} \mathrm{NMR}\left(\mathrm{CDCl}_{3}, 75 \mathrm{MHz}\right) \delta 207.7,172.1$, $142.1,139.5,138.9,138.4,136.6,130.1,129.1,128.2,127.3,125.7,59.1$, 53.0, 40.3, 40.0, 32.1, 16.5, 16.0; HRMS (EI) calcd for $\mathrm{C}_{21} \mathrm{H}_{21} \mathrm{O}_{3}\left[\mathrm{M}-\mathrm{CO}_{2} \mathrm{Me}\right]^{+}$321.1491, found 321.1527.

3-Acetyl-4-(2-ethoxycarbonyl-1-methyl-4-phenylbut-1-en-3-ynyl)cyclopent-3-ene1,1-dicarboxylic acid dimethyl ester (3aj, Scheme 1, $96 \%$ yield, $E: Z=1:>20$ )

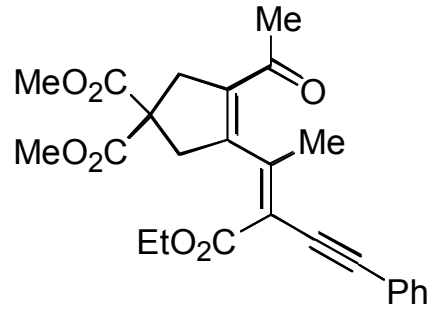

Pale yellow oil; IR (neat) 2954, 1737, 1661, 1255, $759 \mathrm{~cm}^{-1} ;{ }^{1} \mathrm{H}$ NMR $\left(\mathrm{CDCl}_{3}, 300\right.$ $\mathrm{MHz}) \delta 7.50-7.42(\mathrm{~m}, 2 \mathrm{H}), 7.34-7.28(\mathrm{~m}, 3 \mathrm{H}), 4.14(\mathrm{q}, J=7.2 \mathrm{~Hz}, 2 \mathrm{H}), 3.73(\mathrm{~s}, 6 \mathrm{H})$, 3.37-3.32 (m, 4H), $2.28(\mathrm{~s}, 3 \mathrm{H}), 2.16(\mathrm{~s}, 3 \mathrm{H}), 1.24(\mathrm{t}, J=7.2 \mathrm{~Hz}, 3 \mathrm{H}) ;{ }^{13} \mathrm{C} \mathrm{NMR}\left(\mathrm{CDCl}_{3}\right.$, $75 \mathrm{MHz}) \delta 195.1,171.2,163.5,153.9,151.5,132.8,131.4,128.5,128.2,122.6,114.6,96.4$, 84.1, 61.2, 57.2, 52.9, 45.1, 40.9, 28.6, 22.3, 13.9; HRMS (EI) calcd for $\mathrm{C}_{25} \mathrm{H}_{26} \mathrm{O}_{7}[\mathrm{M}]^{+}$ 438.1679 , found 438.1714 .

\section{Rh-Catalyzed Reactions of 1,6-Diynes with Arylketones}

(Table 2 and Schemes 2,3)

General Procedure (Table 2, entry 1). Segphos (9.2 $\mathrm{mg}, 0.015 \mathrm{mmol})$ and $\left[\mathrm{Rh}(\mathrm{cod})_{2}\right] \mathrm{BF}_{4}(6.1 \mathrm{mg}, 0.015 \mathrm{mmol})$ were dissolved in $\mathrm{CH}_{2} \mathrm{Cl}_{2}(2.0 \mathrm{~mL})$, and the mixture was stirred at $\mathrm{rt}$ for $5 \mathrm{~min}$. $\mathrm{H}_{2}$ was introduced to the resulting solution in a Schlenk tube. 
After stirring at $\mathrm{rt}$ for $0.5 \mathrm{~h}$, the resulting solution was concentrated to dryness and dissolved in $\mathrm{CH}_{2} \mathrm{Cl}_{2}(0.5 \mathrm{~mL})$. To this solution was added dropwise a solution of $\mathbf{1 a}$ (70.9 $\mathrm{mg}, 0.300 \mathrm{mmol})$ and $\mathbf{2 k}(360.5 \mathrm{mg}, 3.00 \mathrm{mmol})$ in $\mathrm{CH}_{2} \mathrm{Cl}_{2}$ at $\mathrm{rt}$ and washed remaining substrates away by using $\mathrm{CH}_{2} \mathrm{Cl}_{2}(1.0 \mathrm{~mL})$. The mixture was stirred at $\mathrm{rt}$ for $3 \mathrm{~h}$. The resulting solution was concentrated and purified by silica gel column chromatography $\left(\mathrm{CH}_{2} \mathrm{Cl}_{2}\right.$ :hexane $\left.=1: 1\right)$ followed by preparative TLC (hexane:ethyl acetate $=5: 1$ ), which furnished 5ak (93.6 mg, $0.263 \mathrm{mmol}, 88 \%$ yield) as a pale yellow oil.

3-[1-(2-Acetylphenyl)ethylidene]-4-ethylidenecyclopentane-1,1-dicarboxylic acid dimethyl ester (5ak, Table 2, entry 1, 88\% yield, $E: Z=1:>20$ )

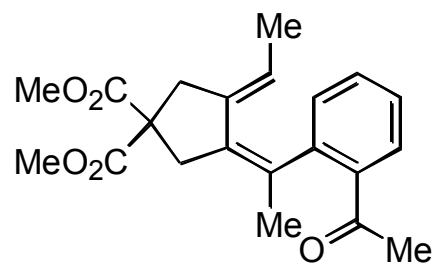

IR (neat) 2954, 1736, 1690, 1253, $733 \mathrm{~cm}^{-1} ;{ }^{1} \mathrm{H}$ NMR $\left(\mathrm{CDCl}_{3}, 300 \mathrm{MHz}\right) \delta 7.63-7.61$ $(\mathrm{m}, 1 \mathrm{H}), 7.47-7.42(\mathrm{~m}, 1 \mathrm{H}), 7.34-7.29(\mathrm{~m}, 1 \mathrm{H}), 7.12-7.09(\mathrm{~m}, 1 \mathrm{H}), 4.55-4.45(\mathrm{~m}, 1 \mathrm{H})$, 3.78-2.74 (m, 6H), $3.12(\mathrm{~s}, 2 \mathrm{H}), 2.94(\mathrm{~s}, 2 \mathrm{H}), 2.42$ (s, 3H), 2.02 (s, 3H), 1.40 (d, J = 7.2 Hz, $3 \mathrm{H}) ;{ }^{13} \mathrm{C} \mathrm{NMR}\left(\mathrm{CDCl}_{3}, 75 \mathrm{MHz}\right) \delta 201.4,172.0,171.8,143.4,138.1,136.3,132.5,131.8$, $129.4,129.3,128.5,126.7,121.1,56.8,52.72,52.68,39.8,38.0,28.7,24.0,15.2$; HRMS (EI) calcd for $\mathrm{C}_{20} \mathrm{H}_{21} \mathrm{O}_{4}[\mathrm{M}-\mathrm{OMe}]^{+} 325.1440$, found 325.1512 .

3-[1-(2-Acetyl-5-methoxyphenyl)ethylidene]-4-ethylidenecyclopentane-1,1dicarboxylic acid dimethyl ester (5al, Table 2, entry 2, 89\% yied, $E: Z=1: 5$ )

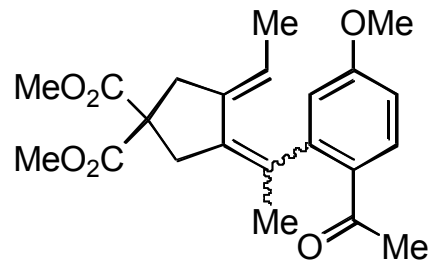

Although crude NMR showed only $E$-isomer, $E / Z$ isomerization occurred during isolation by silica gel chromatography. Pale yellow oil; IR (neat) 2954, 1736, 1681, 1257, $733 \mathrm{~cm}^{-1} ;{ }^{1} \mathrm{H} \mathrm{NMR}\left(\mathrm{CDCl}_{3}, 300 \mathrm{MHz}\right) \delta 7.73(\mathrm{~d}, J=9.0 \mathrm{~Hz}, 1 \mathrm{H}), 6.82(\mathrm{dd}, J=9.0,2.7 \mathrm{~Hz}$, $1 \mathrm{H}), 6.58(\mathrm{~d}, 2.7 \mathrm{~Hz}, 1 \mathrm{H}), 4.62-4.54(\mathrm{~m}, 1 \mathrm{H}), 3.83(\mathrm{~s}, 3 \mathrm{H}), 3.76(\mathrm{~s}, 6 \mathrm{H}), 3.11(\mathrm{~s}, 2 \mathrm{H}), 2.94$ $(\mathrm{s}, 2 \mathrm{H}), 2.40(\mathrm{~s}, 3 \mathrm{H}), 2.01(\mathrm{~s}, 3 \mathrm{H}), 1.42(\mathrm{~d}, J=6.9 \mathrm{~Hz}, 3 \mathrm{H})$; Z-isomer: ${ }^{1} \mathrm{H} \mathrm{NMR}\left(\mathrm{CDCl}_{3}, 300\right.$ MHz) $\delta 7.73(\mathrm{~d}, J=9.0 \mathrm{~Hz}, 1 \mathrm{H}), 6.82(\mathrm{dd}, J=9.0,2.7 \mathrm{~Hz}, 1 \mathrm{H}), 6.61(\mathrm{~d}, \mathrm{~J}=2.7 \mathrm{~Hz}, 1 \mathrm{H})$, 5.93-5.86 (m, 1H), $3.85(\mathrm{~s}, 3 \mathrm{H}), 3.68(\mathrm{~s}, 3 \mathrm{H}), 3.66(\mathrm{~s}, 3 \mathrm{H}), 3.06(\mathrm{~s}, 2 \mathrm{H}), 3.02(\mathrm{~s}, 2 \mathrm{H}), 2.44$ $(\mathrm{s}, 3 \mathrm{H}), 2.16(\mathrm{~s}, 3 \mathrm{H}), 1.80(\mathrm{~d}, J=6.9 \mathrm{~Hz} .3 \mathrm{H}) ;{ }^{13} \mathrm{C} \mathrm{NMR}\left(\mathrm{CDCl}_{3}, 75 \mathrm{MHz}\right) \delta 198.9,172.0$, $171.8,162.2,146.5,137.7,136.2,131.9,131.7,131.6,130.1,130.0,122.4,120.9,114.3$, $111.9,111.8,56.9,56.8,55.2,52.70,52.66,52.6,40.7,39.8,38.1,38.0,28.6,28.4,23.9$, 22.7, 15.4, 15.3; HRMS (EI) calcd for $\mathrm{C}_{22} \mathrm{H}_{26} \mathrm{O}_{6}[\mathrm{M}]^{+}$386.1729, found 386.1675. 
3-Ethylidene-4-[1-(2-propionylphenyl)ethylidene]cyclopentane-1,1-dicarboxylic acid dimethyl ester (5an, Table 2, entry 4, 94\% yield, $E: Z=1: 2$ )

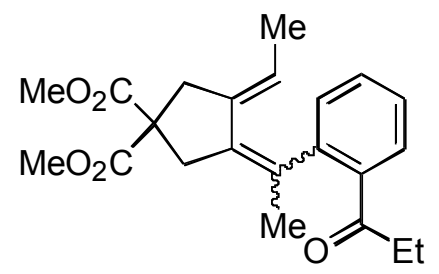

Although crude NMR showed only $E$-isomer, $E / Z$ isomerization occurred during isolation by silica gel chromatography. Yellow oil; IR (neat) 2954, 1737, 1692, 1436, 1255, $648 \mathrm{~cm}^{-1} ;{ }^{1} \mathrm{H}$ NMR $\left(\mathrm{CDCl}_{3}, 300 \mathrm{MHz}\right) \delta 7.59-7.54(\mathrm{~m}, 1 \mathrm{H}), 7.47-7.39(\mathrm{~m}, 1 \mathrm{H}), 7.34-7.27$, $(\mathrm{m}, 1 \mathrm{H}), 7.14-7.08(\mathrm{~m}, 1 \mathrm{H}), 4.55-4.45(\mathrm{~m}, 1 \mathrm{H}), 3.77(\mathrm{~s}, 3 \mathrm{H}), 3.76(\mathrm{~s}, 3 \mathrm{H}), 3.10(\mathrm{~s}, 2 \mathrm{H})$, $2.92(\mathrm{~s}, 2 \mathrm{H}), 2.87-2.69(\mathrm{~m}, 2 \mathrm{H}), 2.01(\mathrm{~s}, 3 \mathrm{H}), 1.40(\mathrm{~d}, J=6.9 \mathrm{~Hz}, 3 \mathrm{H}), 1.10(\mathrm{t}, J=7.2 \mathrm{~Hz}$, $3 \mathrm{H})$; vinyl, $\mathrm{Me}$, and Et protons of $E$-isomer: ${ }^{1} \mathrm{H} \mathrm{NMR}\left(\mathrm{CDCl}_{3}, 300 \mathrm{MHz}\right) \delta 5.92-5.83(\mathrm{~m}$, $1 \mathrm{H}), 2.87-2.69(\mathrm{~m}, 2 \mathrm{H}), 2.16(\mathrm{~s}, 3 \mathrm{H}), 1.79(\mathrm{~d}, J=7.2 \mathrm{~Hz}, 3 \mathrm{H}), 1.12(\mathrm{t}, J=7.2 \mathrm{~Hz}, 3 \mathrm{H}) ;{ }^{13} \mathrm{C}$ NMR $\left(\mathrm{CDCl}_{3}, 75 \mathrm{MHz}\right) \delta 205.2,204.6,172.1,171.8,144.3,143.2,138.5,138.4,137.7$, $136.4,133.6,132.2,131.5,131.1,130.9,129.6,129.5,129.0,128.1,126.73,126.66,122.6$, 121.0, 57.0, 56.8, 52.8, 52.7, 52.6, 40.9, 39.8, 38.1, 38.0, 34.5, 34.1, 24.2, 23.1, 15.5, 15.3, 8.5, 8.4; HRMS (EI) calcd for $\mathrm{C}_{22} \mathrm{H}_{26} \mathrm{O}_{5}[\mathrm{M}]^{+} 370.1780$, found 370.1761 .

3-Ethylidene-4-[1-(2-isobutyrylphenyl)ethylidene]cyclopentane-1,1-dicarboxylic acid dimethyl ester (5ao, Table 2, entry 5, 82\% yield, $E: Z=1:>20$ )

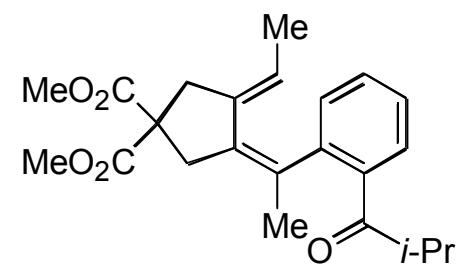

Pale yellow oil; IR (neat) 2972, 1737, 1690, 1253, $734 \mathrm{~cm}^{-1} ;{ }^{1} \mathrm{H}$ NMR $\left(\mathrm{CDCl}_{3}, 300\right.$ $\mathrm{MHz}) \delta 7.56-7.50(\mathrm{~m}, 1 \mathrm{H}), 7.46-7.38(\mathrm{~m}, 1 \mathrm{H}), 7.34-7.27(\mathrm{~m}, 1 \mathrm{H}), 7.14-7.09(\mathrm{~m}, 1 \mathrm{H})$, 4.58-4.47 (m, 1H), 3.77-3.75 (m, 6H), 3.28 (sept, $J=6.9 \mathrm{~Hz}, 1 \mathrm{H}), 3.17-3.04(\mathrm{~m}, 2 \mathrm{H})$, 2.98-2.84 (m, 2H), $2.00(\mathrm{~s}, 3 \mathrm{H}), 1.40$ (d, $J=7.2 \mathrm{~Hz}, 3 \mathrm{H}), 1.07$ (dd, $J=13.2,7.2 \mathrm{~Hz}, 6 \mathrm{H})$; ${ }^{13} \mathrm{C} \mathrm{NMR}\left(\mathrm{CDCl}_{3}, 75 \mathrm{MHz}\right) \delta 208.3,172.1,143.4,137.9,136.3,132.0,131.3,129.7,129.5$, 128.4, 126.6, 121.1, 56.8, 52.7, 39.8, 38.13, 38.11, 24.1, 19.1, 18.7, 15.2; HRMS (EI) calcd for $\mathrm{C}_{23} \mathrm{H}_{28} \mathrm{O}_{5}[\mathrm{M}]^{+}$384.1937, found 384.1959.

3-[1-(2-Benzoylphenyl)ethylidene]-4-ethylidenecyclopentane-1,1-dicarboxylic acid dimethyl ester (5ap, Table 2, entry 6, $97 \%$ yield, $E: Z=1: 9$ ) 


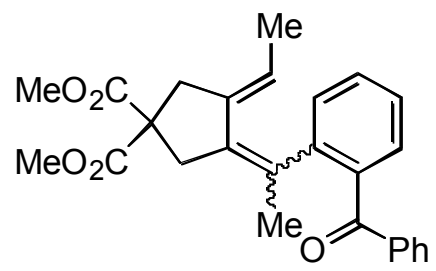

Although crude NMR showed only $E$-isomer, $E / Z$ isomerization occurred during isolation by silica gel chromatography. Pale yellow oil; IR (neat) 2954, 1736, 1665, 1263, $733 \mathrm{~cm}^{-1} ;{ }^{1} \mathrm{H}$ NMR $\left(\mathrm{CDCl}_{3}, 300 \mathrm{MHz}\right) \delta$ 7.66-7.63 (m, 2H), 7.55-7.19 (m, 7H), 4.73-4.62 $(\mathrm{m}, 1 \mathrm{H}), 3.68(\mathrm{~s}, 3 \mathrm{H}), 3.66(\mathrm{~s}, 3 \mathrm{H}), 3.02-2.86(\mathrm{~m}, 2 \mathrm{H}), 2.68-2.52(\mathrm{~m}, 2 \mathrm{H}), 1.88(\mathrm{~s}, 3 \mathrm{H})$, $1.42(\mathrm{~d}, J=6.9 \mathrm{~Hz}, 3 \mathrm{H})$; vinyl and Me protons of $E$-isomer: ${ }^{1} \mathrm{H}$ NMR $\left(\mathrm{CDCl}_{3}, 300 \mathrm{MHz}\right) \delta$ $5.40-5.32(\mathrm{~m}, 1 \mathrm{H}), 1.92(\mathrm{~s}, 3 \mathrm{H}), 1.65(\mathrm{~d}, J=6.9 \mathrm{~Hz}, 3 \mathrm{H}) ;{ }^{13} \mathrm{C} \mathrm{NMR}\left(\mathrm{CDCl}_{3}, 75 \mathrm{MHz}\right) \delta$ 197.2, 172.0, 171.6, 143.9, 137.8, 137.7, 134.0, 132.3, 130.1, 129.6, 129.4, 129.3, 129.2, $128.3,127.9,127.8,126.0,121.4,56.6,52.59,52.56,39.3,37.5,23.9,15.2$; HRMS (EI) calcd for $\mathrm{C}_{25} \mathrm{H}_{23} \mathrm{O}_{4}[\mathrm{M}-\mathrm{OMe}]^{+}$387.1596, found 387.1581.

1-[2-\{1-(4-Ethylidene-1-(toluene-4-sulfonyl)pyrrolidin-3ylidene)ethyl $\}$ phenyl]ethanone (5bk, Table 2, entry $7,84 \%$ yield, $E: Z=1:>10$ )

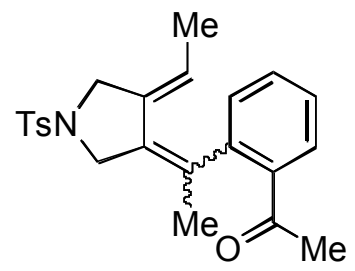

Although crude NMR showed only $E$-isomer, $E / Z$ isomerization occurred during isolation by silica gel chromatography. Pale yellow solid; Mp 73.8-74.5 ${ }^{\circ} \mathrm{C}$; IR (neat) 2983 , $1686,1346,1164,670 \mathrm{~cm}^{-1} ;{ }^{1} \mathrm{H}$ NMR $\left(\mathrm{CDCl}_{3}, 300 \mathrm{MHz}\right) \delta 7.79-7.67(\mathrm{~m}, 3 \mathrm{H}), 7.46(\mathrm{dt}, J=$ 7.5, $1.5 \mathrm{~Hz}, 1 \mathrm{H}), 7.38-7.32(\mathrm{~m}, 3 \mathrm{H}), 7.01(\mathrm{dd}, J=7.8,1.2 \mathrm{~Hz}, 1 \mathrm{H}), 4.43-4.34(\mathrm{~m}, 1 \mathrm{H})$, 4.19-4.13 (m, 1H), 4.01-3.92 (m, 2H), 3.84-3.77 (m, 1H), 2.46 (s, 3H), 2.40 (s, 3H), 1.92 $(\mathrm{s}, 3 \mathrm{H}), 1.33(\mathrm{~d}, J=7.2 \mathrm{~Hz}, 3 \mathrm{H})$; vinyl and Me protons of $E$-isomer: ${ }^{1} \mathrm{H}$ NMR $\left(\mathrm{CDCl}_{3}, 300\right.$ MHz) $\delta 5.90-5.82(\mathrm{~m}, 1 \mathrm{H}), 2.43(\mathrm{~s}, 3 \mathrm{H}), 2.42(\mathrm{~s}, 3 \mathrm{H}), 2.11(\mathrm{~s}, 3 \mathrm{H}), 1.73(\mathrm{~d}, J=7.2 \mathrm{~Hz}$, $3 \mathrm{H}) ;{ }^{13} \mathrm{C}$ NMR $\left(\mathrm{CDCl}_{3}, 75 \mathrm{MHz}\right) \delta 200.1,143.7,142.7,137.1,133.4,133.0,132.6,130.8$, 129.7, 129.6, 129.3, 129.2, 128.2, 127.8, 127.3, 122.4, 120.5, 52.7, 51.5, 28.6, 23.7, 21.5, 15.3; HRMS (EI) calcd for $\mathrm{C}_{23} \mathrm{H}_{25} \mathrm{NO}_{3} \mathrm{~S}[\mathrm{M}]^{+} 395.1555$, found 395.1541 .

1-\{2-[1-(4-Ethylidenedihydrofuran-3-ylidene)ethyl]-4-methoxyphenyl\}ethanone (5dl, Table 2, entry $8,69 \%$ yield, $E: Z=1:>10$ ) 


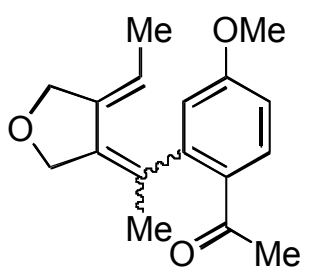

Although crude NMR showed only $E$-isomer, $E / Z$ isomerization occurred during isolation by silica gel chromatography. Colorless oil; IR (neat) 2940, 1682, 1596, 1131, 731 $\mathrm{cm}^{-1} ;{ }^{1} \mathrm{H} \mathrm{NMR}\left(\mathrm{CDCl}_{3}, 300 \mathrm{MHz}\right) \delta 7.81(\mathrm{~d}, J=8.7 \mathrm{~Hz}, 1 \mathrm{H}), 6.86(\mathrm{dd}, J=8.7,2.7 \mathrm{~Hz}, 1 \mathrm{H})$, $6.65(\mathrm{~d}, J=2.7 \mathrm{~Hz}, 1 \mathrm{H}), 4.64-4.55(\mathrm{~m}, 2 \mathrm{H}), 4.54-4.39(\mathrm{~m}, 3 \mathrm{H}), 3.85(\mathrm{~s}, 3 \mathrm{H}), 2.46(\mathrm{~s}, 3 \mathrm{H})$, $1.94(\mathrm{~s}, 3 \mathrm{H}), 1.38(\mathrm{~d}, J=6.9 \mathrm{~Hz}, 3 \mathrm{H})$; phenyl, vinyl, and Me protons of $E$-isomer: ${ }^{1} \mathrm{H}$ NMR $\left(\mathrm{CDCl}_{3}, 300 \mathrm{MHz}\right) \delta 7.74(\mathrm{~d}, J=8.7 \mathrm{~Hz}, 1 \mathrm{H}), 6.83(\mathrm{dd}, J=2.7 \mathrm{~Hz}, 1 \mathrm{H}), 6.62(\mathrm{~d}, J=2.7 \mathrm{~Hz}$, $1 \mathrm{H}), 5.96-5.89(\mathrm{~m}, 1 \mathrm{H}), 3.84(\mathrm{~s}, 3 \mathrm{H}), 2.20(\mathrm{~s}, 3 \mathrm{H}), 1.76(\mathrm{~d}, J=6.9 \mathrm{~Hz}, 3 \mathrm{H}),{ }^{13} \mathrm{C}$ NMR $\left(\mathrm{CDCl}_{3}, 75 \mathrm{MHz}\right) \delta 198.4,162.9,145.8,136.3,132.1,132.0,130.1,129.6,128.2,119.7$, 117.6, 114.3, 112.4, 105.6, 72.8, 71.8, 55.4, 28.5, 23.4, 22.1, 15.4; HRMS (EI) calcd for $\mathrm{C}_{17} \mathrm{H}_{20} \mathrm{O}_{3}[\mathrm{M}]^{+}$272.1421, found 272.1356. yield)

1-[2-\{1-(4-Ethylfuran-3-yl)ethyl\}-4-methoxyphenyl]ethanone (6, Scheme 2, 71\%<smiles>CCc1cocc1C(c1cc(OC)ccc1C(C)=O)[N+](=O)[O-]</smiles>

Colorless oil; IR (neat) 2966, 1674, 1604, 1046, $742 \mathrm{~cm}^{-1} ;{ }^{1} \mathrm{H}$ NMR $\left(\mathrm{CDCl}_{3}, 300 \mathrm{MHz}\right)$ $\delta 7.75-7.67(\mathrm{~m}, 1 \mathrm{H}), 7.30-7.27(\mathrm{~m}, 1 \mathrm{H}), 7.15-7.11(\mathrm{~m}, 1 \mathrm{H}), 6.70-6.60(\mathrm{~m}, 2 \mathrm{H}), 4.94(\mathrm{dq}, J$ $=7.2,1.2 \mathrm{~Hz}, 1 \mathrm{H}), 3.75(\mathrm{~s}, 3 \mathrm{H}), 2.58(\mathrm{~s}, 3 \mathrm{H}), 2.12-1.98(\mathrm{~m}, 1 \mathrm{H}), 1.94-1.81(\mathrm{~m}, 1 \mathrm{H}), 1.48$ $(\mathrm{d}, J=6.9 \mathrm{~Hz}, 3 \mathrm{H}), 0.96(\mathrm{t}, J=7.5 \mathrm{~Hz}, 3 \mathrm{H}) ;{ }^{13} \mathrm{C} \mathrm{NMR}\left(\mathrm{CDCl}_{3}, 75 \mathrm{MHz}\right) \delta 200.3,162.1$, 149.5, 139.6, 139.1, 132.0, 129.64, 129.61, 126.6, 114.6, 110.2, 55.1, 31.0, 29.6, 22.0, 16.7, 13.0; HRMS (EI) calcd for $\mathrm{C}_{17} \mathrm{H}_{20} \mathrm{O}_{3}[\mathrm{M}]^{+}$272.1421, found 272.1356.

3-[1-(2-Acetyl-4-methoxyphenyl)ethylidene]-4-ethylidenecyclopentane-1,1dicarboxylic acid diethyl ester (5aq, Scheme 3, 95\% yield, $E: Z=1: 6$ )

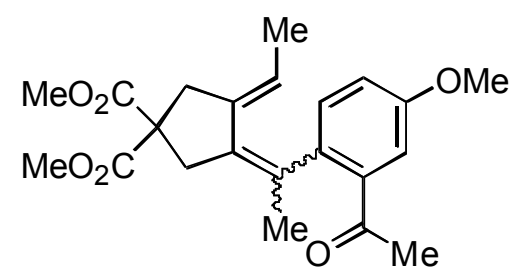

Although crude NMR showed only $E$-isomer, $E / Z$ isomerization occurred during isolation by silica gel chromatography. Pale yellow oil; IR (neat) 2954, 1736, 1691, 1283, 
$734 \mathrm{~cm}^{-1} ;{ }^{1} \mathrm{H}$ NMR $\left(\mathrm{CDCl}_{3}, 300 \mathrm{MHz}\right) \delta 7.15-7.11(\mathrm{~m}, 1 \mathrm{H}), 6.99-6.96(\mathrm{~m}, 2 \mathrm{H}), 4.62-4.53$ $(\mathrm{m}, 1 \mathrm{H}), 3.82(\mathrm{~s}, 3 \mathrm{H}), 3.74(\mathrm{~s}, 6 \mathrm{H}), 3.06(\mathrm{~s}, 2 \mathrm{H}), 2.92(\mathrm{~s}, 2 \mathrm{H}), 2.38(\mathrm{~s}, 3 \mathrm{H}), 1.97(\mathrm{~s}, 3 \mathrm{H})$, $1.41(\mathrm{~d}, J=6.9 \mathrm{~Hz}, 3 \mathrm{H})$; vinyl, $\mathrm{Me}$, and OMe protons of $E$-isomer: ${ }^{1} \mathrm{H} \mathrm{NMR}\left(\mathrm{CDCl}_{3}, 300\right.$ MHz) $\delta 5.88-5.79(\mathrm{~m}, 1 \mathrm{H}), 3.64(\mathrm{~s}, 3 \mathrm{H}), 2.40(\mathrm{~s}, 3 \mathrm{H}), 2.14(\mathrm{~s}, 3 \mathrm{H}), 1.77(\mathrm{~d}, J=6.9 \mathrm{~Hz}$, $3 \mathrm{H}) ;{ }^{13} \mathrm{C} \mathrm{NMR}\left(\mathrm{CDCl}_{3}, 75 \mathrm{MHz}\right) \delta 201.5,172.1,171.9,158.1,139.2,136.4,135.8,133.0$, 130.6, 129.0, 121.2, 118.1, 117.7, 113.2, 56.8, 55.3, 52.79, 52.77, 40.0, 38.2, 28.8, 24.4, 23.3, 15.4; HRMS (EI) calcd for $\mathrm{C}_{22} \mathrm{H}_{26} \mathrm{O}_{6}[\mathrm{M}]^{+} 386.1729$, found 386.1735.

\section{3-[1-(2-Acetyl-4,6-dimethoxyphenyl)ethylidene]-4-ethylidenecyclopentane-1,1-} dicarboxylic acid diethyl ester (5ar, Scheme 3, 44\% yield, $E: Z=1:>20,39 \%$ ee)

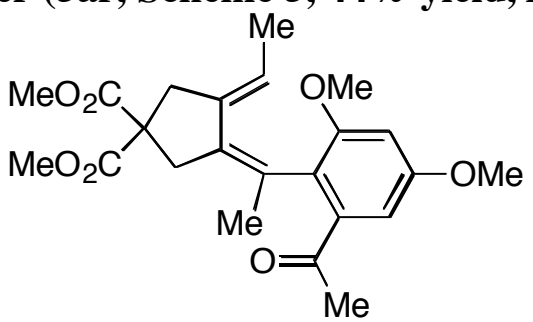

Pale yellow oil; $[\alpha]^{25}+5.47\left(c 1.18, \mathrm{CHCl}_{3}, 39 \%\right.$ ee) IR (neat) $2954,1736,1695,1206$, $733 \mathrm{~cm}^{-1} ;{ }^{1} \mathrm{H} \mathrm{NMR}\left(\mathrm{CDCl}_{3}, 300 \mathrm{MHz}\right) \delta 6.59(\mathrm{~d}, J=2.4 \mathrm{~Hz}, 1 \mathrm{H}), 6.57(\mathrm{~d}, J=2.4 \mathrm{~Hz}, 1 \mathrm{H})$, $4.68-4.57(\mathrm{~m}, 1 \mathrm{H}), 3.83(\mathrm{~s}, 3 \mathrm{H}), 3.76(\mathrm{~s}, 3 \mathrm{H}), 3.75(\mathrm{~s}, 3 \mathrm{H}), 3.74(\mathrm{~s}, 3 \mathrm{H}), 3.15(\mathrm{~d}, J=16.2$ $\mathrm{Hz}, 1 \mathrm{H}), 3.04(\mathrm{~d}, J=16.2 \mathrm{~Hz}, 1 \mathrm{H}), 2.91(\mathrm{~s}, 2 \mathrm{H}), 2.33(\mathrm{~s}, 3 \mathrm{H}), 1.97(\mathrm{~s}, 3 \mathrm{H}), 1.44$ (d. $J=6.9$ $\mathrm{Hz}, 3 \mathrm{H}) ;{ }^{13} \mathrm{C} \mathrm{NMR}\left(\mathrm{CDCl}_{3}, 75 \mathrm{MHz}\right) \delta 202.5,172.1,172.0,159.3,157.6,141.2,136.6$, 134.3, 125.3, 123.8, 120.1, 103.3, 101.6, 56.8, 56.0, 55.4, 52.9, 52.8, 40.0, 38.2, 29.3, 23.1, 15.4; HRMS (EI) calcd for $\mathrm{C}_{22} \mathrm{H}_{25} \mathrm{O}_{6}[\mathrm{M}-\mathrm{OMe}]^{+}$385.1651, found 385.1643. CHIRALPAK AD-H, hexane:2-PrOH = 95:5, $1.0 \mathrm{~mL} / \mathrm{min}$, retention times: $12.7 \mathrm{~min}$ (major isomer) and 14.6 min (minor isomer).

3-Acetyl-4-[2-(3,5-dimethoxyphenyl)-1-methylpropenyl]cyclopent-3-ene-1,1dicarboxylic acid dimethyl ester (3ar, Scheme 3, 30\% yield, $E: Z=2: 1$ )<smiles>COc1cc(OC)cc(C(C)=C(C)C2=C(C(C)=O)CC(C(C)=O)(C(C)=O)C2)c1</smiles>

Pale yellow oil; IR (neat) 2954, 1735, 1659, 1591, $704 \mathrm{~cm}^{-1} ;{ }^{1} \mathrm{H}$ NMR $\left(\mathrm{CDCl}_{3}, 300\right.$ $\mathrm{MHz}) \delta 6.73(\mathrm{t}, J=2.4 \mathrm{~Hz}, 1 \mathrm{H}), 6.28(\mathrm{~d}, J=2.4 \mathrm{~Hz}, 2 \mathrm{H}), 3.79(\mathrm{~s}, 6 \mathrm{H}), 3.76(\mathrm{~s}, 6 \mathrm{H})$, 3.40-3.36 (m, 2H), 3.35-3.31 (m, 2H), $2.33(\mathrm{~s}, 3 \mathrm{H}), 1.90-1.85(\mathrm{~m}, 3 \mathrm{H}), 1.78-1.72(\mathrm{~m}, 3 \mathrm{H})$; $Z$-isomer: ${ }^{1} \mathrm{H} \mathrm{NMR}\left(\mathrm{CDCl}_{3}, 300 \mathrm{MHz}\right) \delta 6.28(\mathrm{t}, J=2.1 \mathrm{~Hz}, 1 \mathrm{H}), 6.20(\mathrm{~d}, J=2.1 \mathrm{~Hz}, 2 \mathrm{H})$, $3.71(\mathrm{~s}, 6 \mathrm{H}), 3.63(\mathrm{~s}, 6 \mathrm{H}), 3.18-3.14(\mathrm{~m}, 2 \mathrm{H}), 3.05-3.00(\mathrm{~m}, 2 \mathrm{H}), 2.26(\mathrm{~s}, 3 \mathrm{H}), 2.06-2.02$ 
$(\mathrm{m}, 3 \mathrm{H}), 1.90-1.83(\mathrm{~m}, 3 \mathrm{H}) ;{ }^{13} \mathrm{C} \mathrm{NMR}\left(\mathrm{CDCl}_{3}, 75 \mathrm{MHz}\right) \delta 196.4,196.3,171.9,171.5$, $160.7,160.2$, 154.3, 153.8, 145.3, 144.6, 134.9, 134.7, 134.0, 127.7, 127.6, 105.9, 105.2, 99.2, 98.3, 56.3, 56.1, 55.3, 55.1, 53.0, 52.8, 46.0, 41.0, 28.4, 28.0, 21.7, 20.6, 19.1, 19.0; HRMS (EI) calcd for $\mathrm{C}_{22} \mathrm{H}_{25} \mathrm{O}_{6}[\mathrm{M}-\mathrm{OMe}]^{+} 385.1651$, found 385.1643.

\section{References}

(1) Duong, H. A.; Cross, M. J.; Louie, J. J. Am. Chem. Soc. 2004, 126, 11438.

(2) Tanaka, K.; Suzuki, N.; Nishida, G. Eur. J. Org. Chem. 2006, 3917.

(3) Tanaka, K.; Takeishi, K.; Noguchi, K. J. Am. Chem. Soc. 2006, 128, 4586.

(4) Tekevac, T. N.; Zuo, G.; Simon, K.; Louie, J. J. Org. Chem. 2006, 71, 5834.

(5) Creary, X. J. Org. Chem. 1987, 52, 5026.

(6) Journet, M; Cai, D; DiMichele, L. M; Larsen, R. D. Tetrahedoron Lett. 1998, 39, 6427.

(7) Guo, M.; Li, D; Zhang, Z. J. Org. Chem. 2003, 68, 10172.

(8) Takahashi, T.; Li, Y.; Ito, T.; Xu, F.; Nakajima, K.; Liu, Y. J. Am. Chem. Soc. 2002, 124, 1144. 
3-Acetyl-4-(2,2-bisethoxycarbonyl-1-methylvinyl)cyclopent-3-ene-1,1-dicarboxylic acid dimethyl ester (3aa)
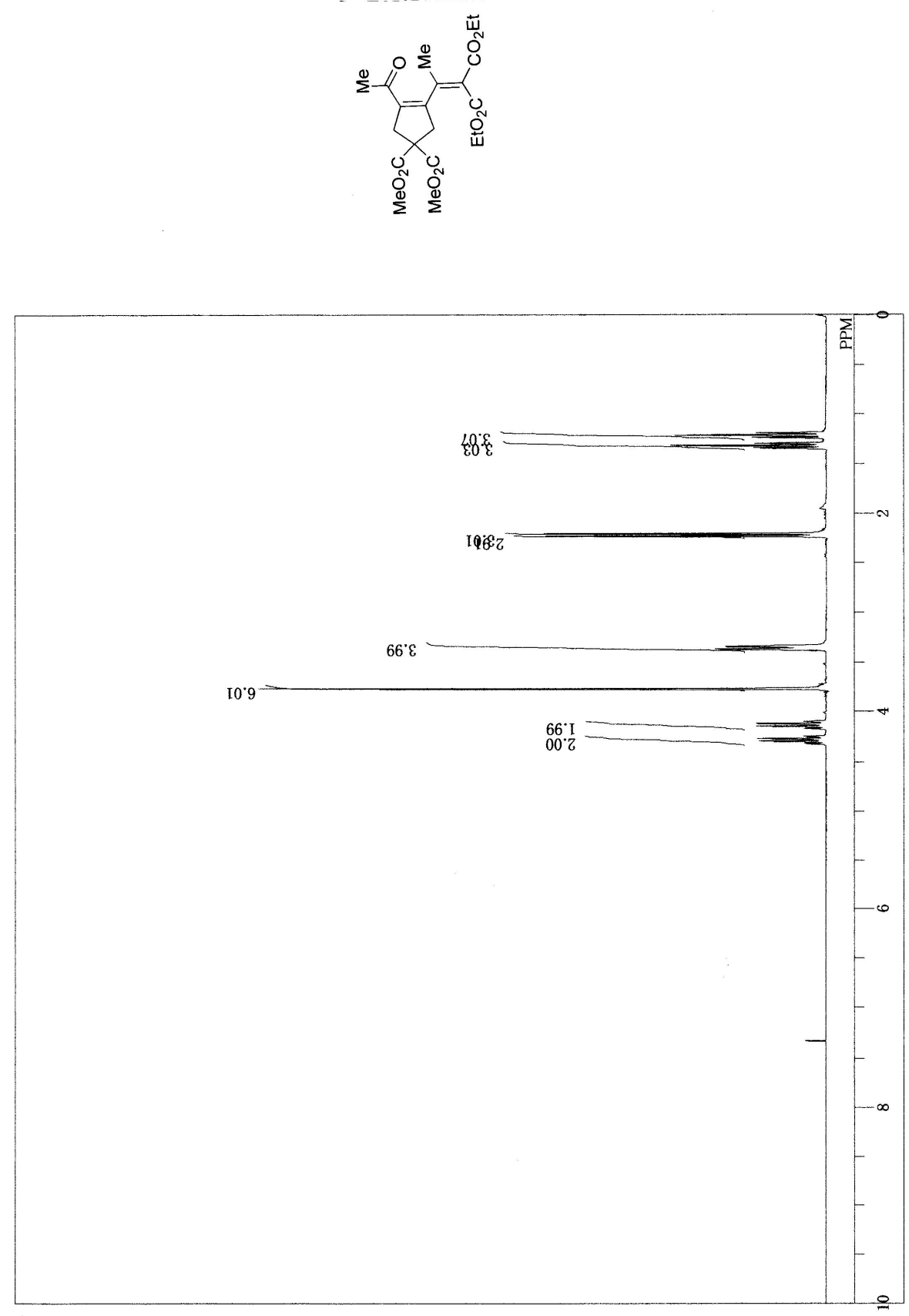
3-Acetyl-4-(2-ethoxycarbonyl-1-methyl-2-phenylvinyl)cyclopent-3-ene-1,1dicarboxylic acid dimethylester (3ab, $E: Z=1: 2)$
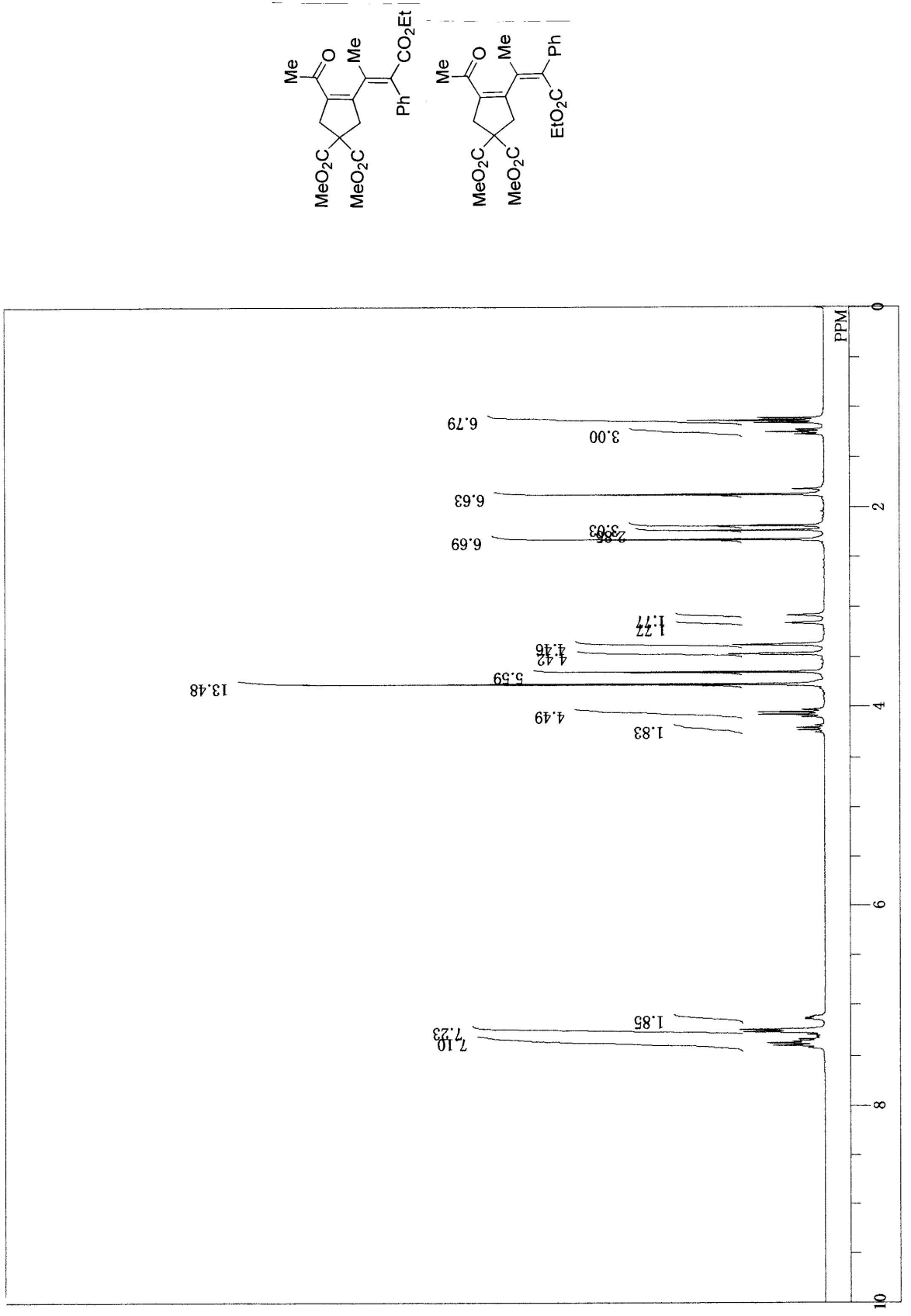
3-Acetyl-4-(2-ethoxycarbonyl-1-methylpropenyl)cyclopent-3-ene-1,1-dicarboxylic acid dimethyl ester (3ac, $E: Z=6: 1$ )
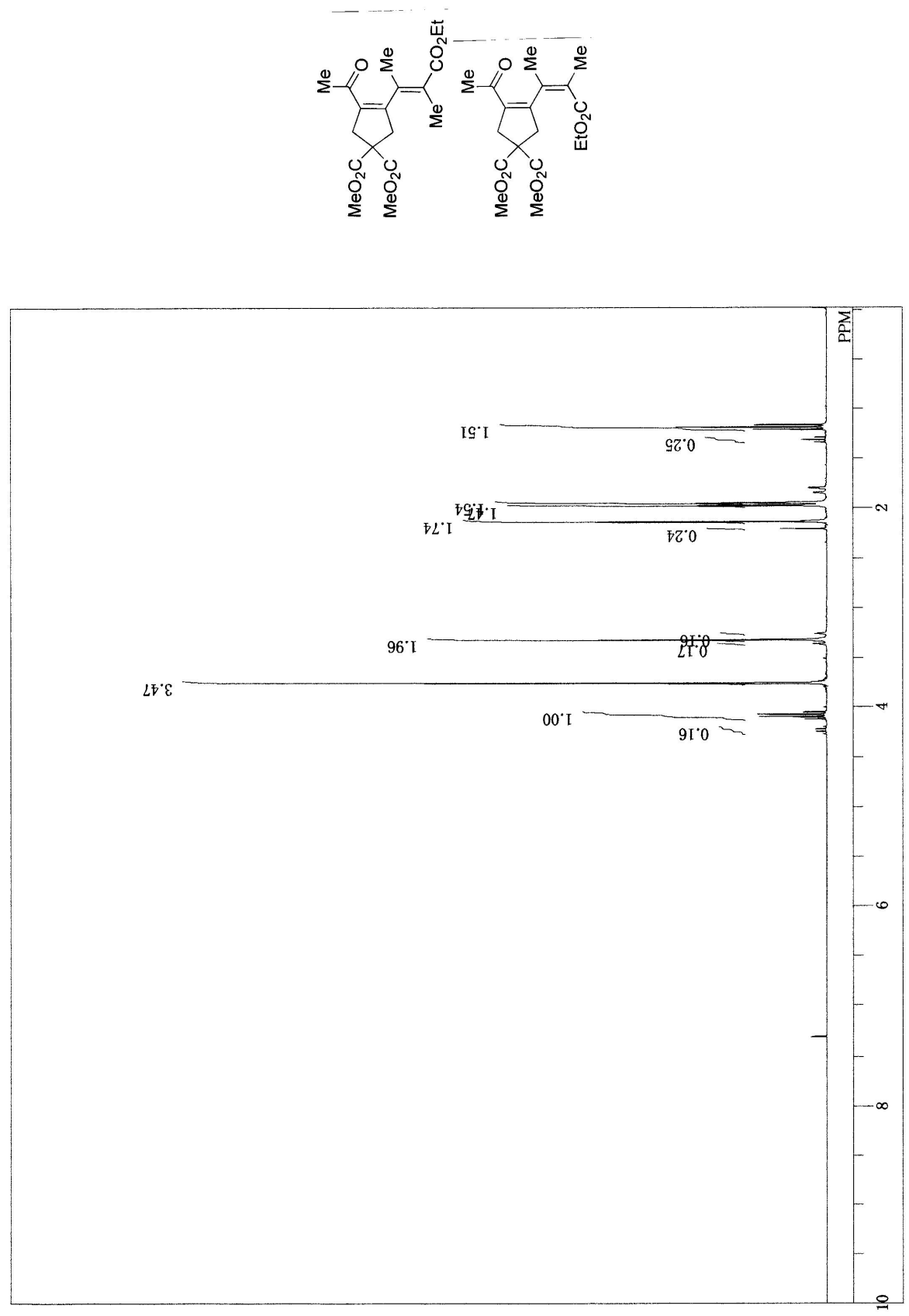
3-Acetyl-4-(1-methyl-2-phenylvinyl)cyclopent-3-ene-1,1-dicarboxylic acid dimethyl ester (3ad, $E: Z=9: 1)$
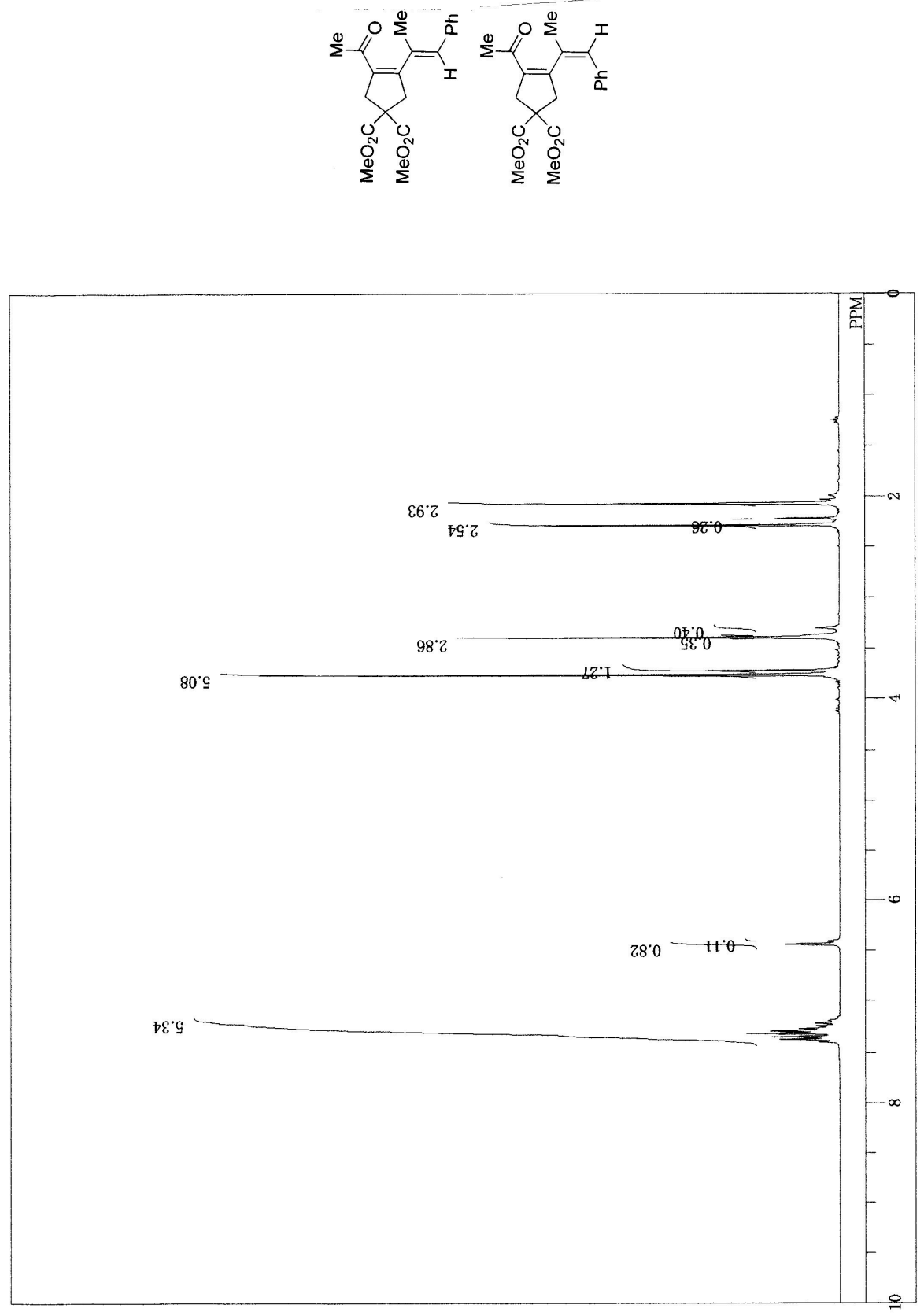
3-Acetyl-4-(1-methylbuta-1,3-dienyl)cyclopent-3-ene-1,1-dicarboxylic acid diethyl ester (3ae, $E: Z=>20: 1)$
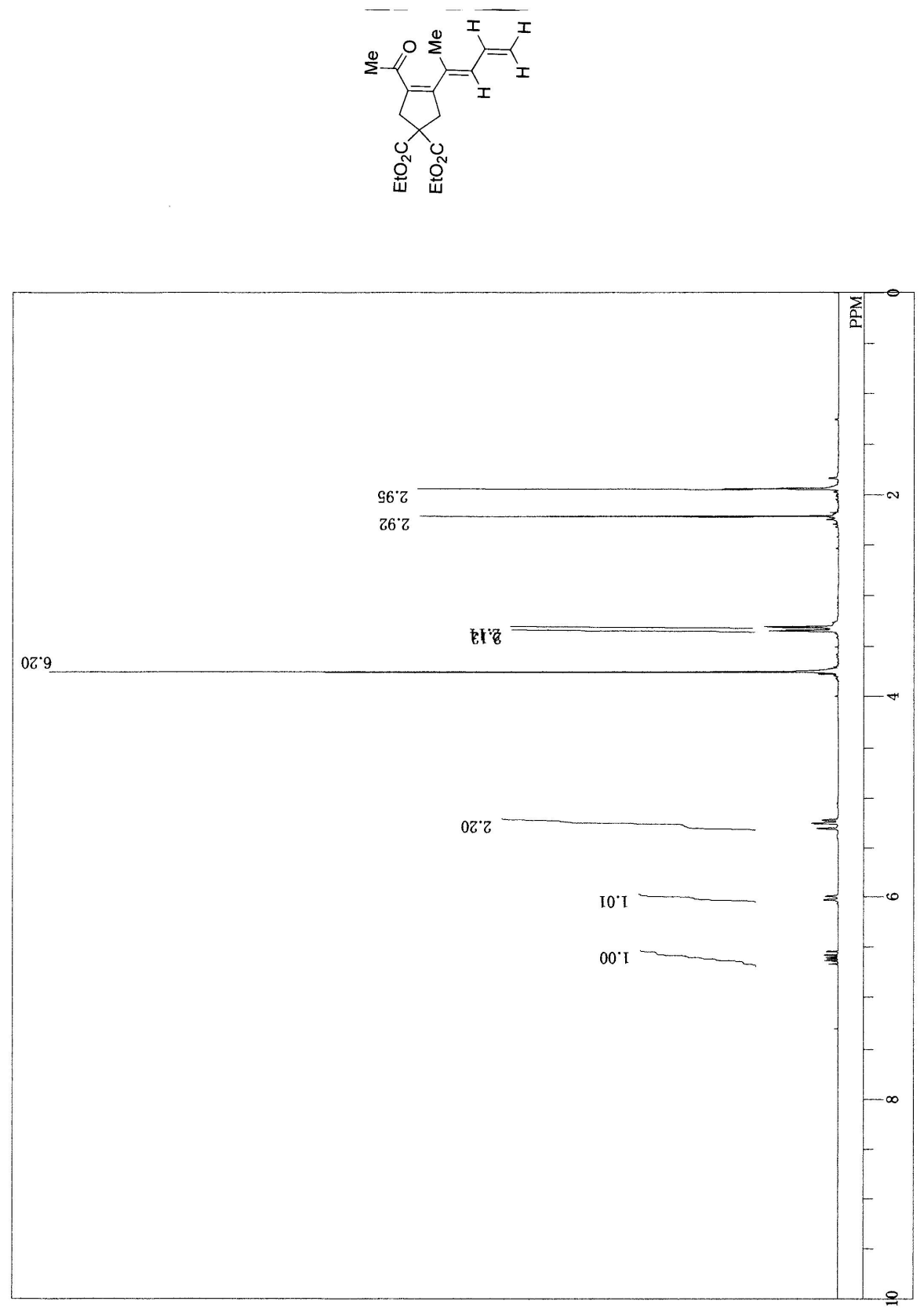
3-Acetyl-4-(1,2-dimethylpropenyl)cyclopent-3-ene-1,1-dicarboxylic acid dimethyl ester (3af) and 1,3,3,4-tetramethyl-3,5-dihydro-7H-cyclopenta[c]pyran-6,6-dicarboxylic acid dimethyl ester (3af, dienone:ether $=3: 1$ )
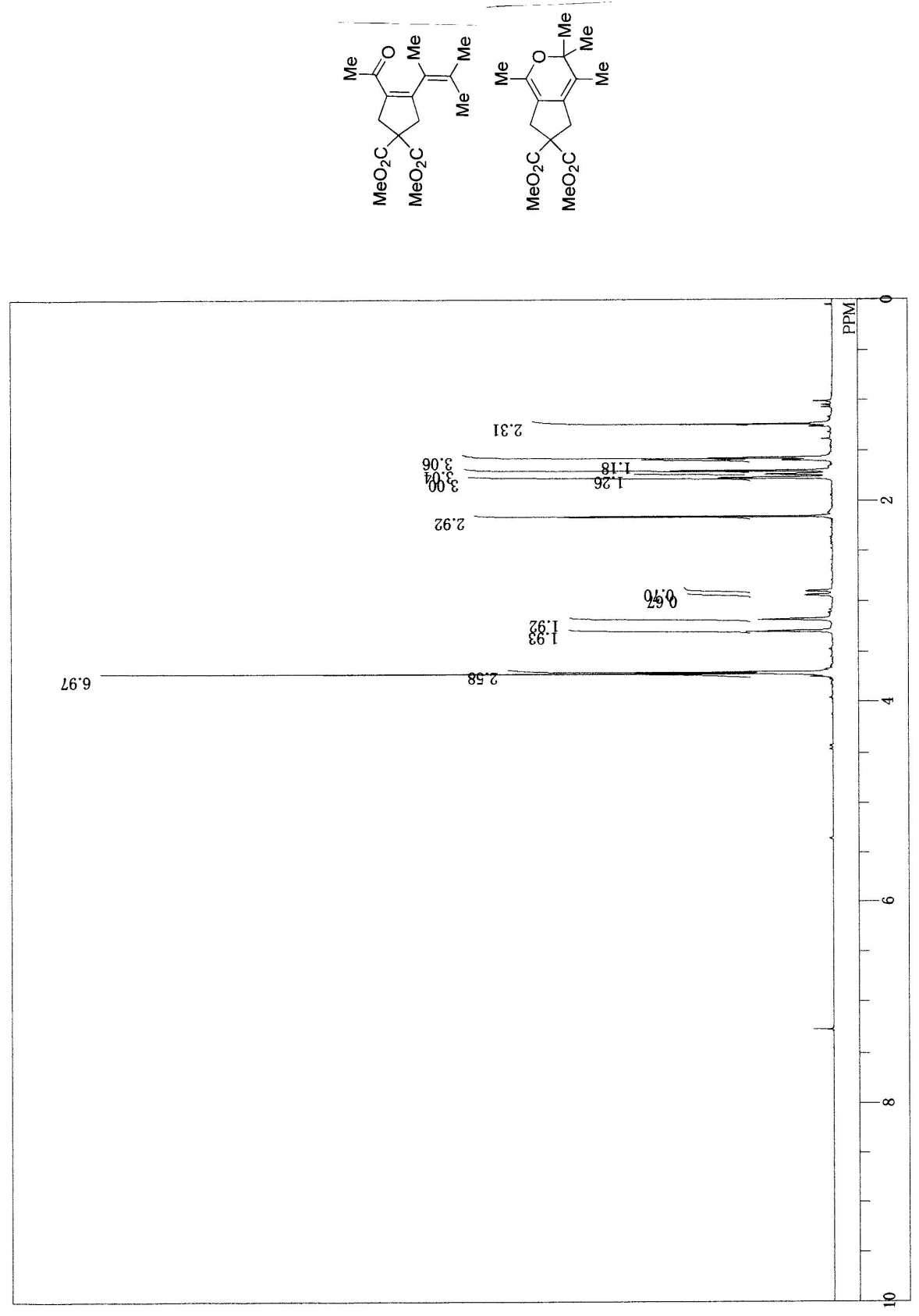
2-[1-\{4-Acetyl-1-(toluene-4-sulfonyl)-2,5-dihydro-1H-pyrrol-3-yl\}ethylidene]malonic acid diethyl ester (3ba)
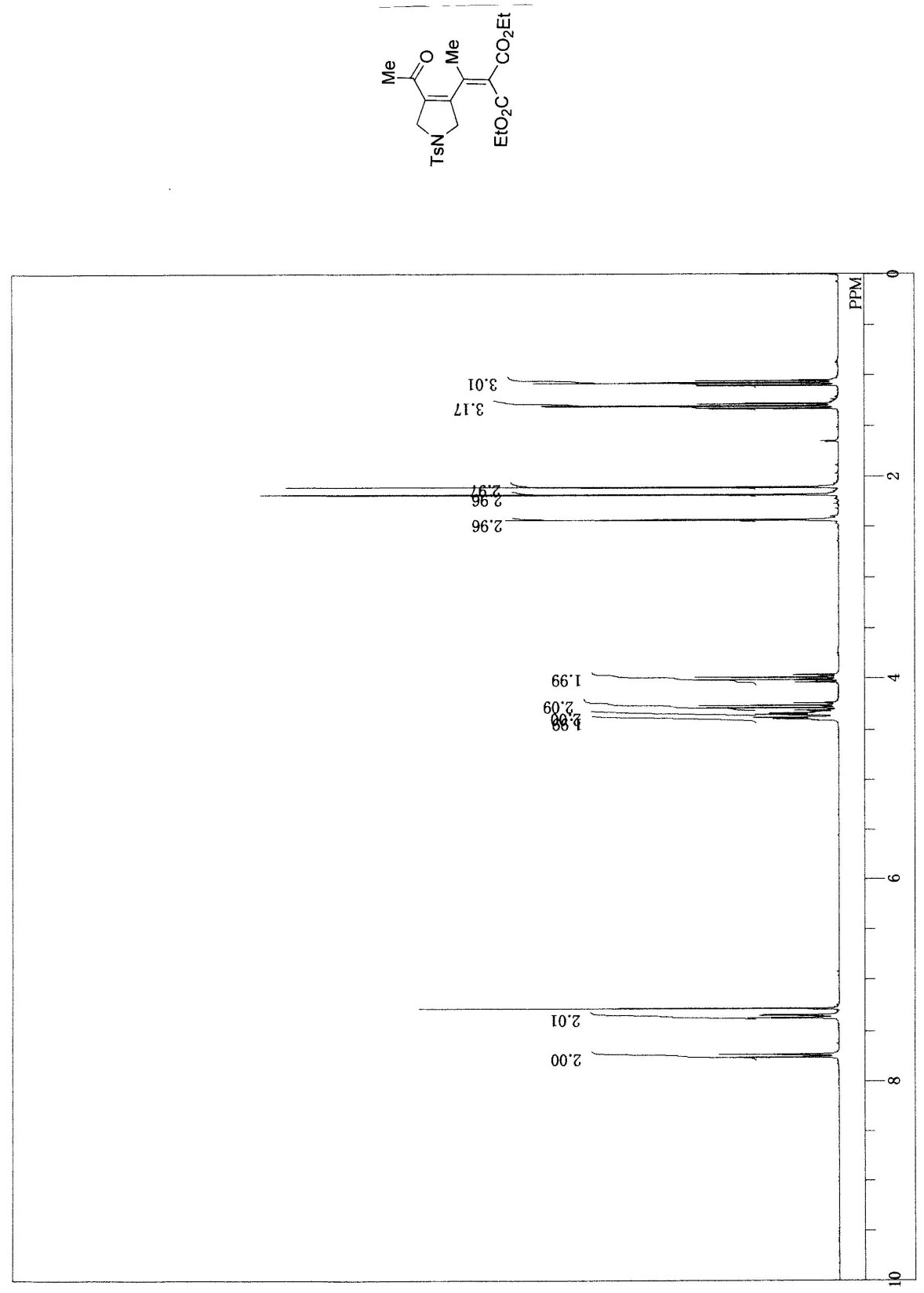
2-[1-(4-Propionyl-2,5-dihydrofuran-3-yl)propylidene]malonic acid diethyl ester (3ca)
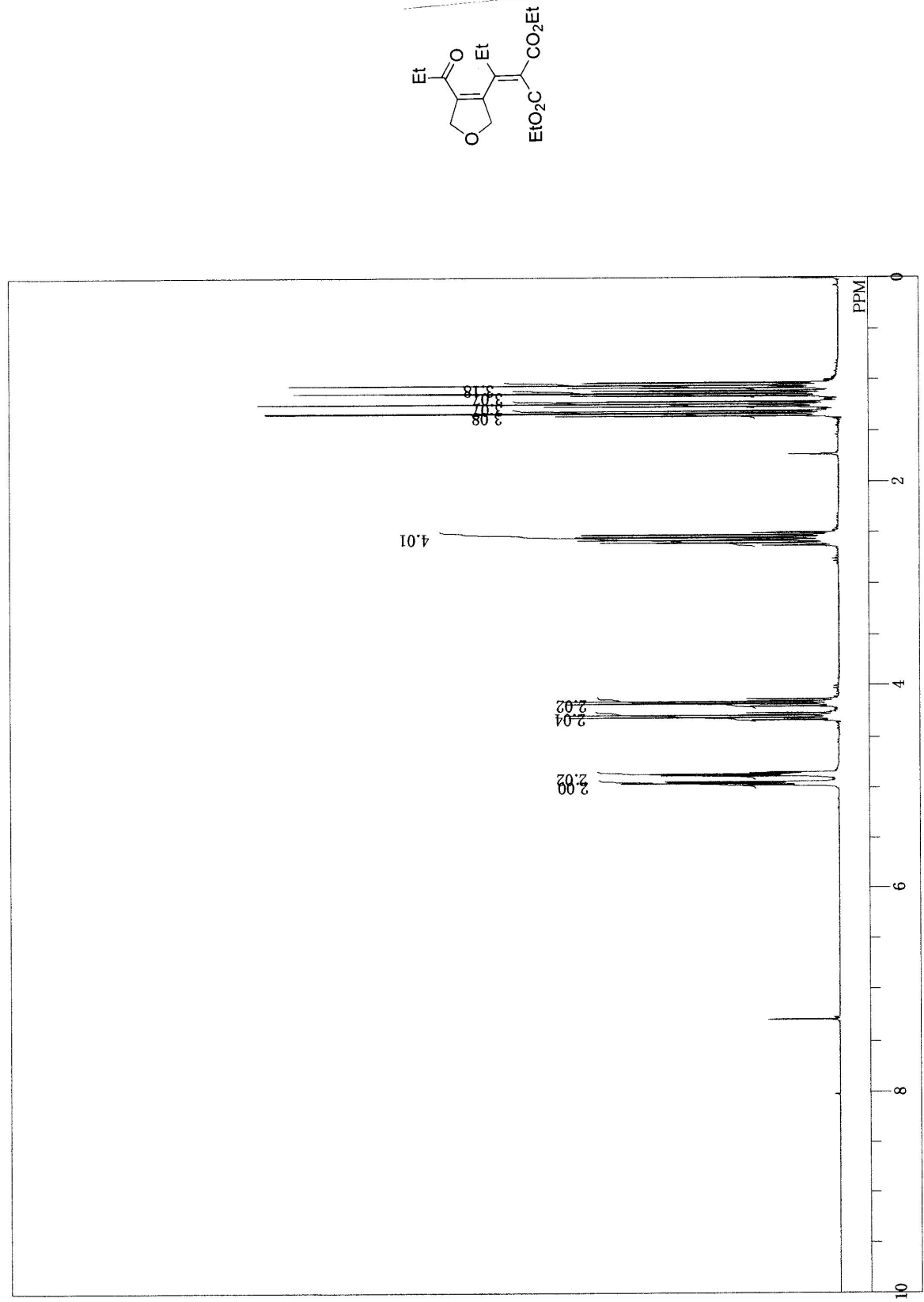
3-Acetyl-4-(1-methyl-4-phenylbut-1-en-3-ynyl)cyclopent-3-ene-1,1-dicarboxylic acid dimethyl ester (3ah, $E: Z=>20: 1$ )
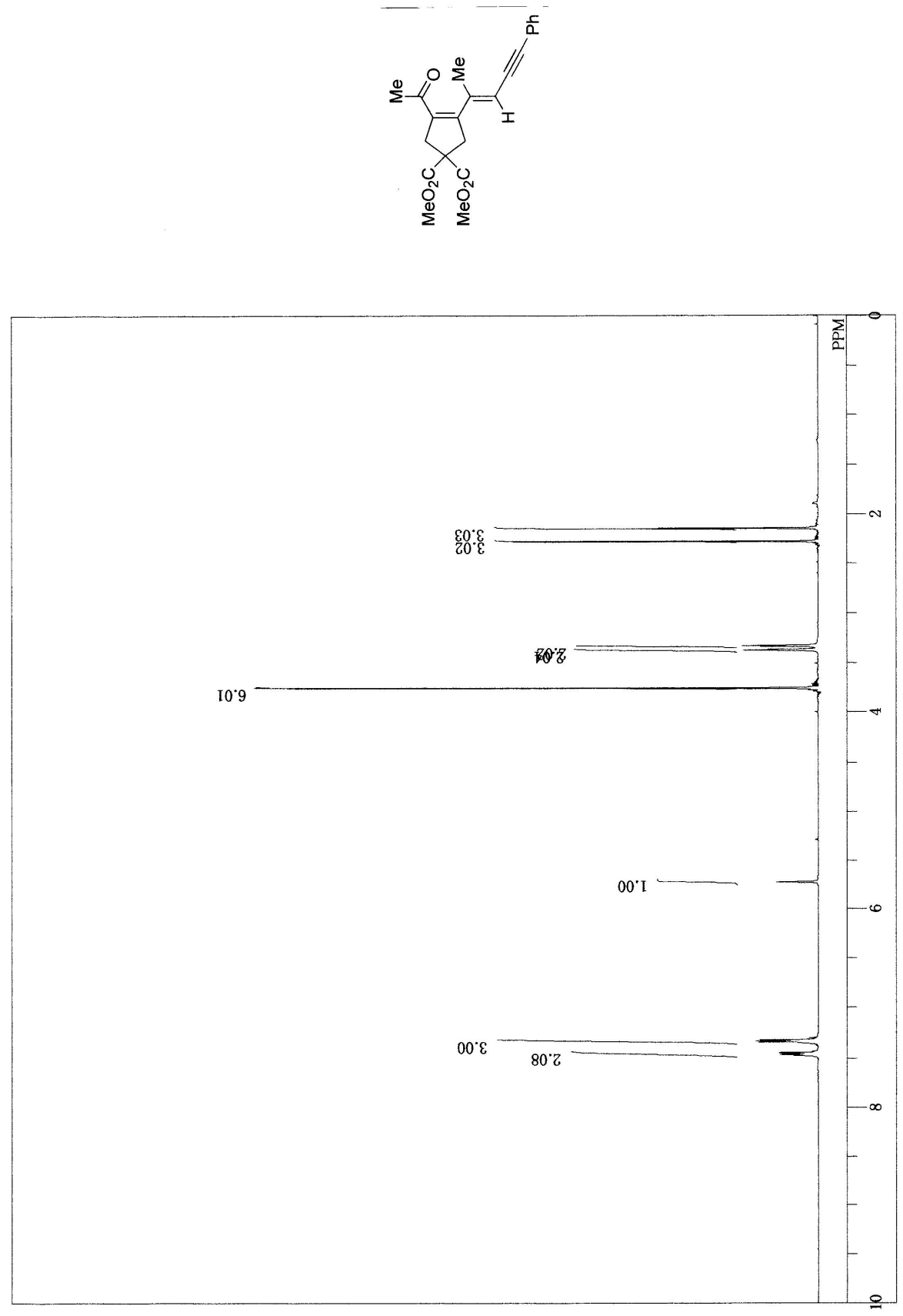
5-Acetyl-4,7-dimethyl-6-phenylindan-2,2-dicarboxylic acid dimethyl ester (4)
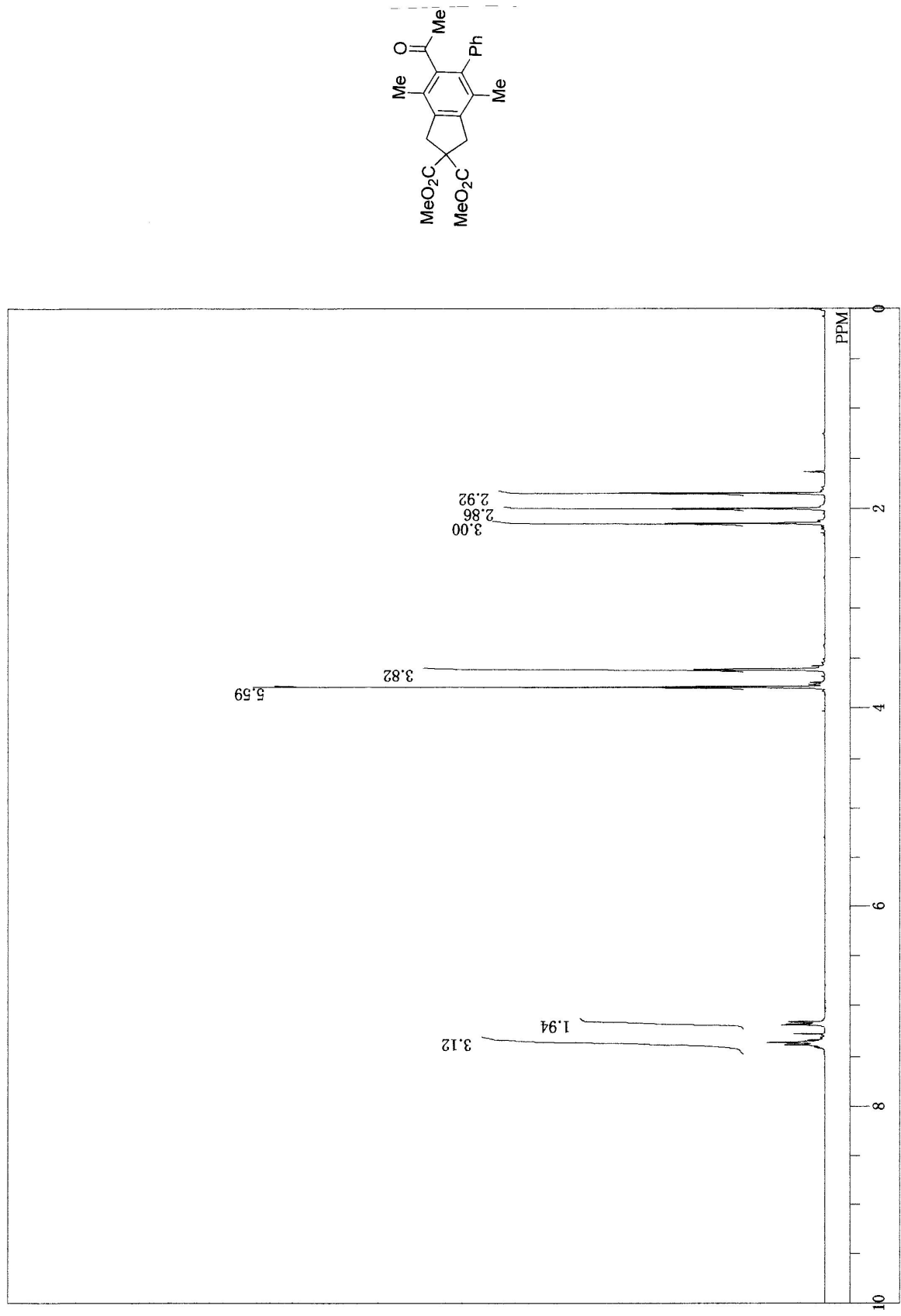
3-Acetyl-4-(2-ethoxycarbonyl-1-methyl-4-phenylbut-1-en-3-ynyl)cyclopent-3-ene-1,1dicarboxylic acid dimethyl ester (3aj, $E: Z=1:>20)$
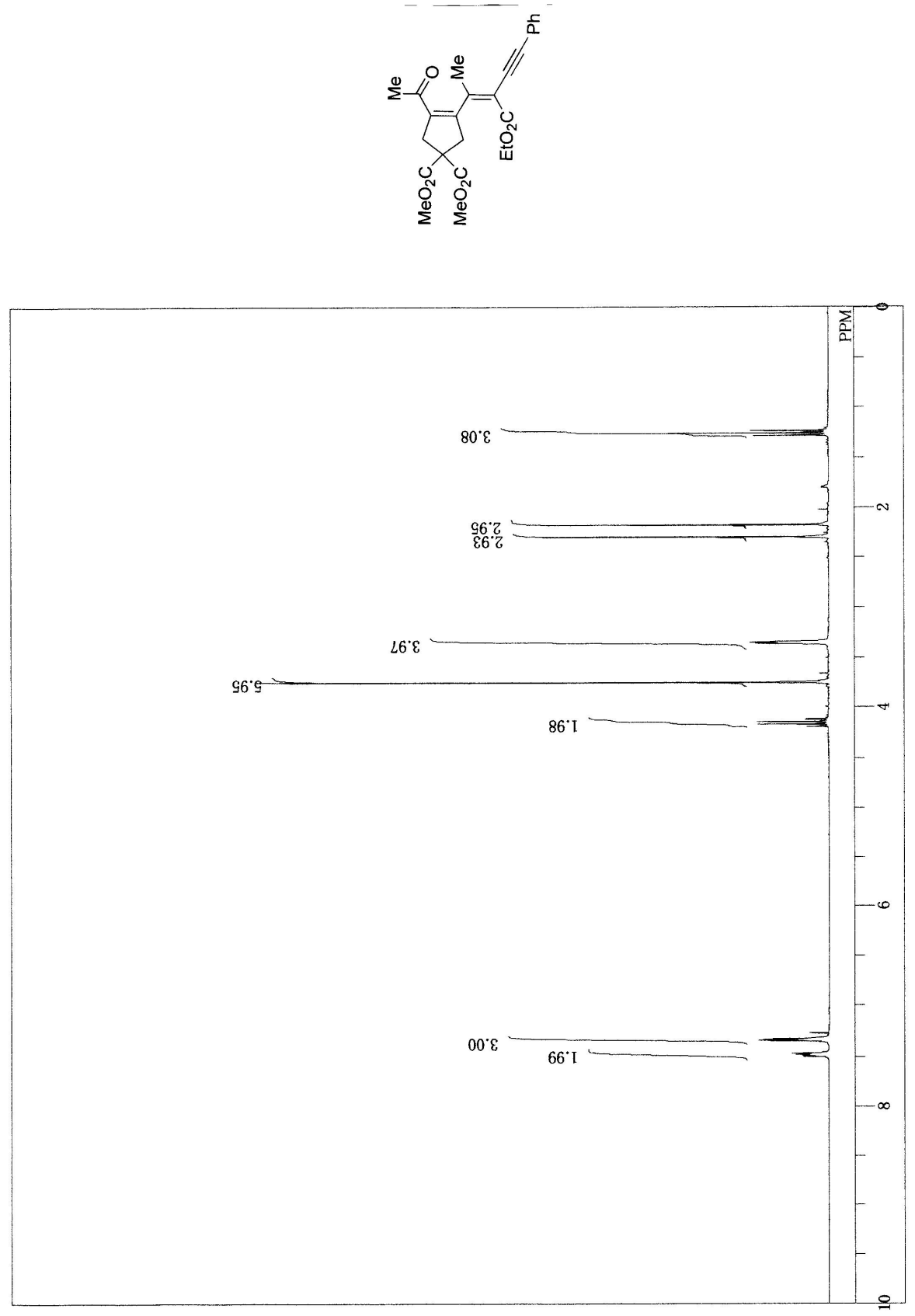

dimethyl ester (5ak, $E: Z=>20: 1)$
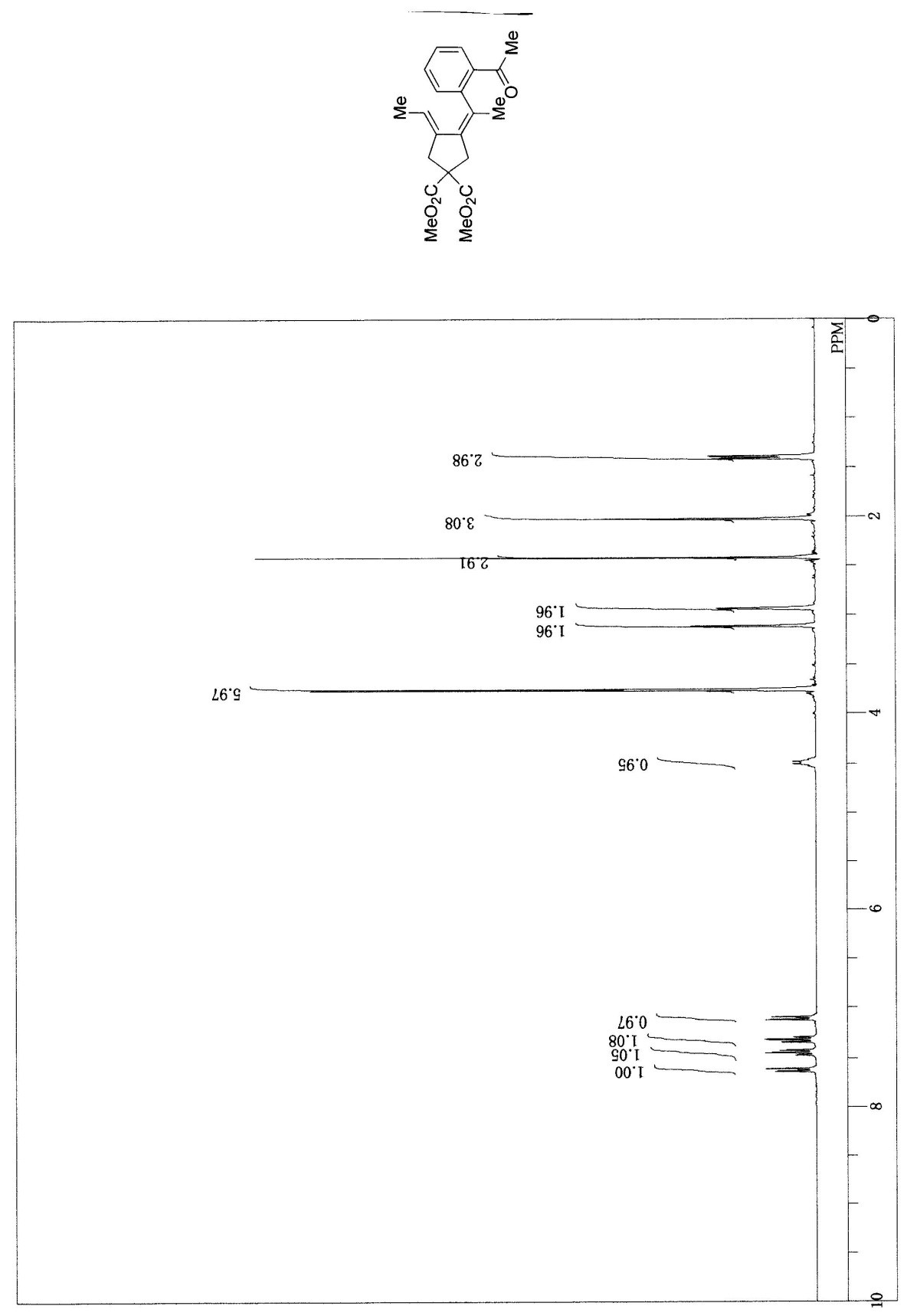
3-[1-(2-Acetyl-5-methoxyphenyl)ethylidene]-4-ethylidenecyclopentane-1,1dicarboxylic acid dimethyl ester (5al, $E: Z=5: 1)$
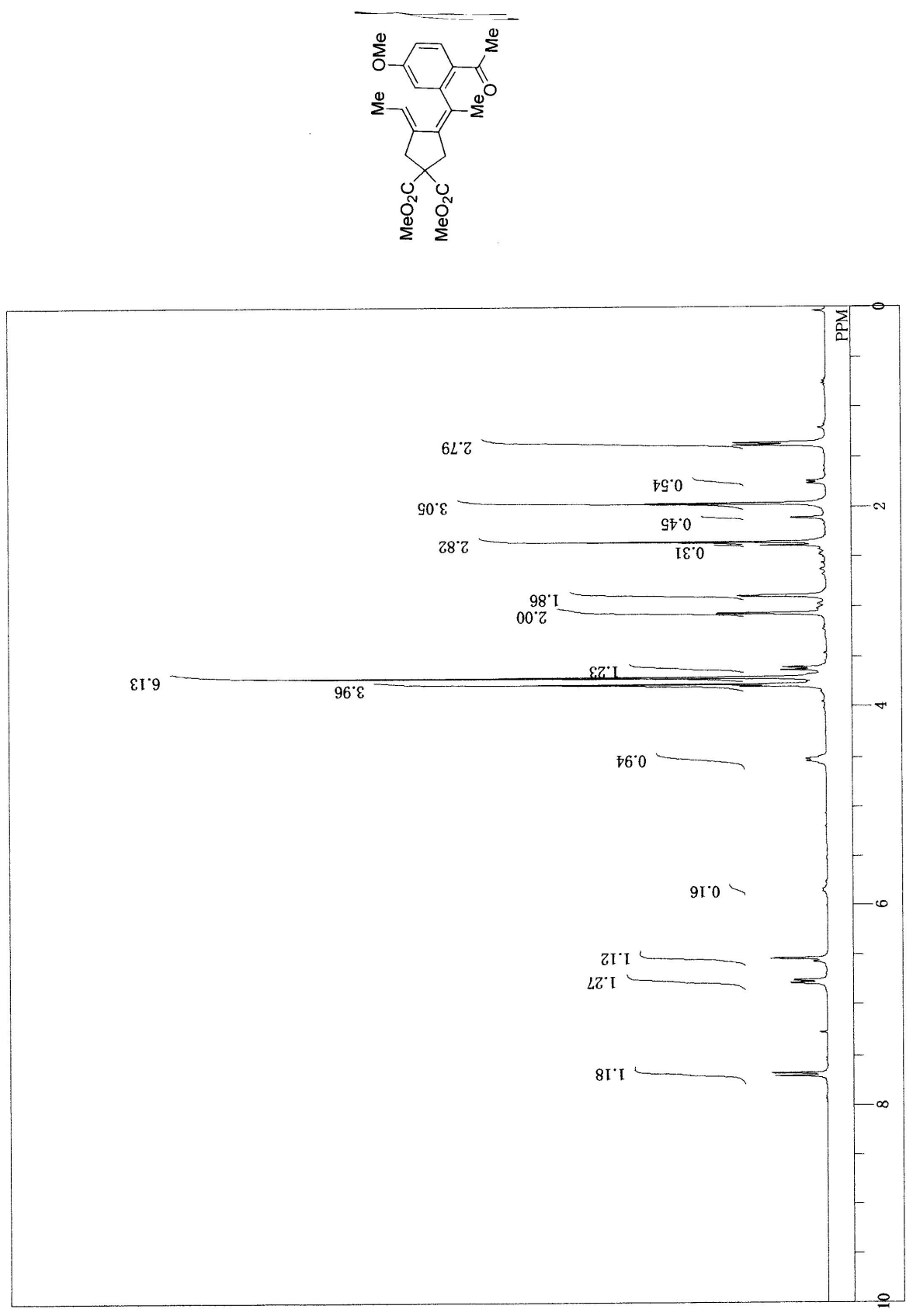
3-Ethylidene-4-[1-(2-propionylphenyl)ethylidene]cyclopentane-1,1-dicarboxylic acid dimethyl ester (5an, $E: Z=2: 1$ )
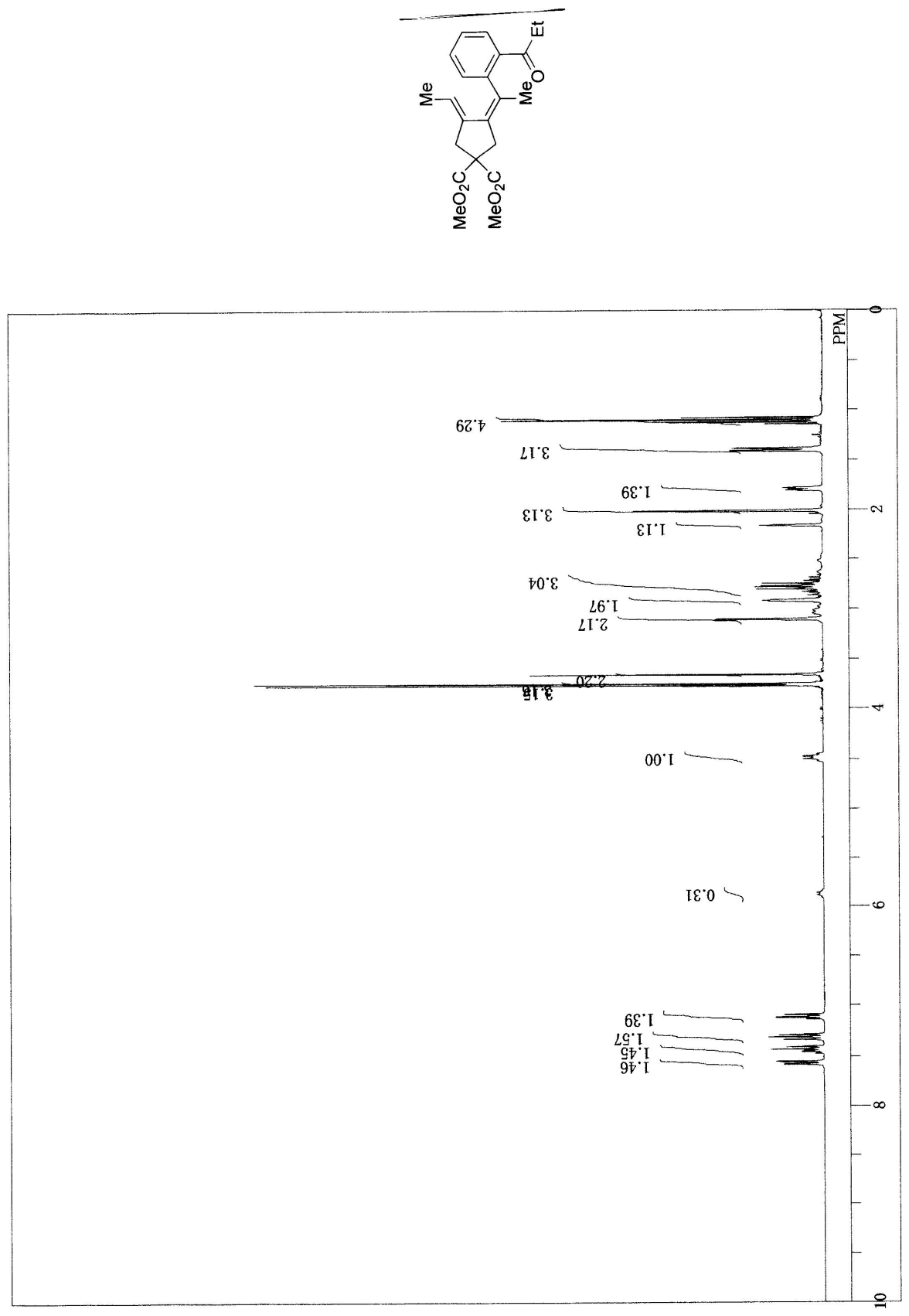
3-Ethylidene-4-[1-(2-isobutyrylphenyl)ethylidene]cyclopentane-1,1-dicarboxylic acid dimethyl ester $(5 \mathbf{a o}, E: Z=>20: 1)$
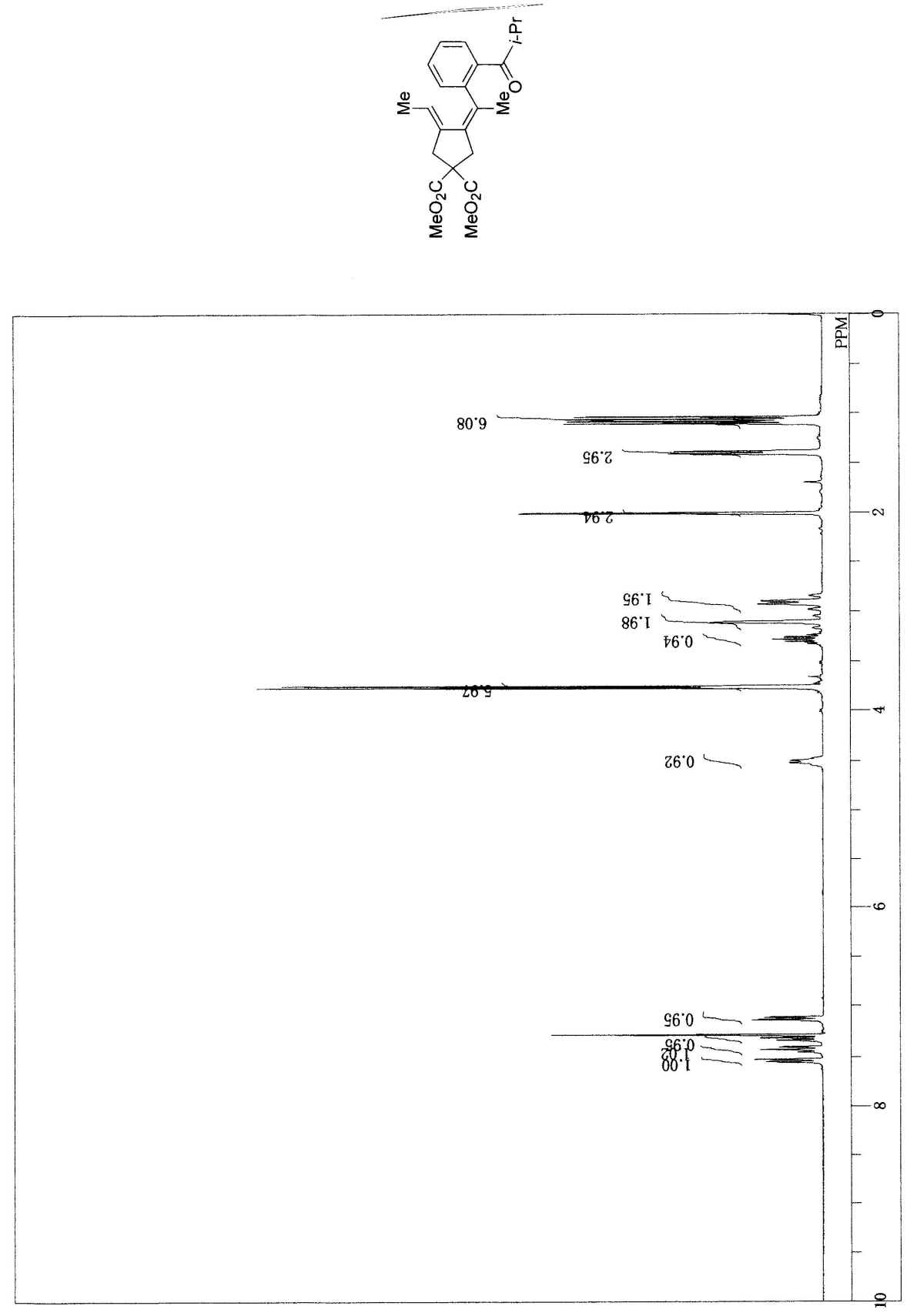

dimethyl ester (5ap, $E: Z=4: 1$ )
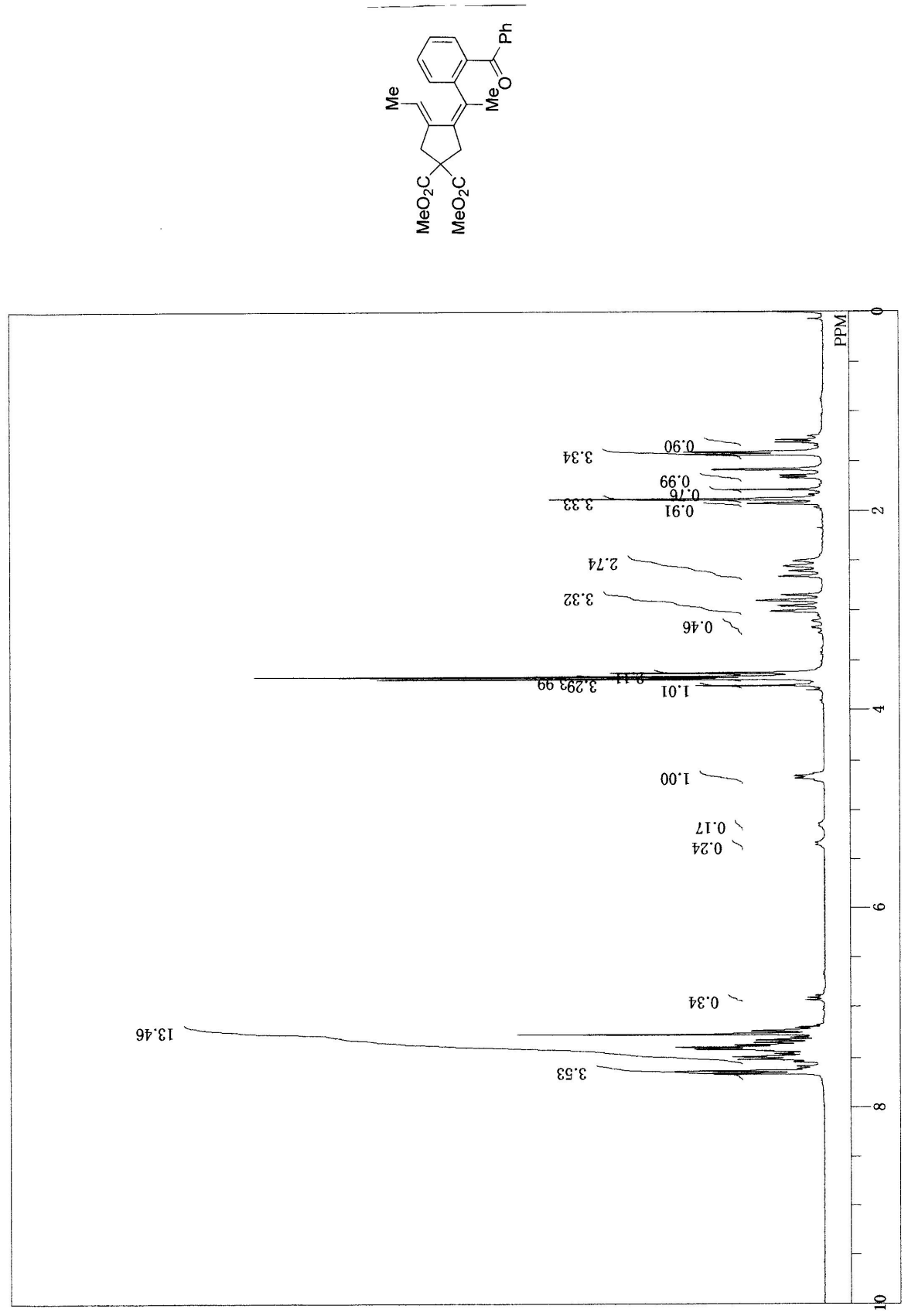


\section{1-[2-\{1-(4-Ethylidene-1-(toluene-4-sulfonyl)pyrrolidin-3-}

ylidene)ethyl $\}$ phenyl]ethanone $(5 \mathrm{bk}, E: Z=>20: 1)$
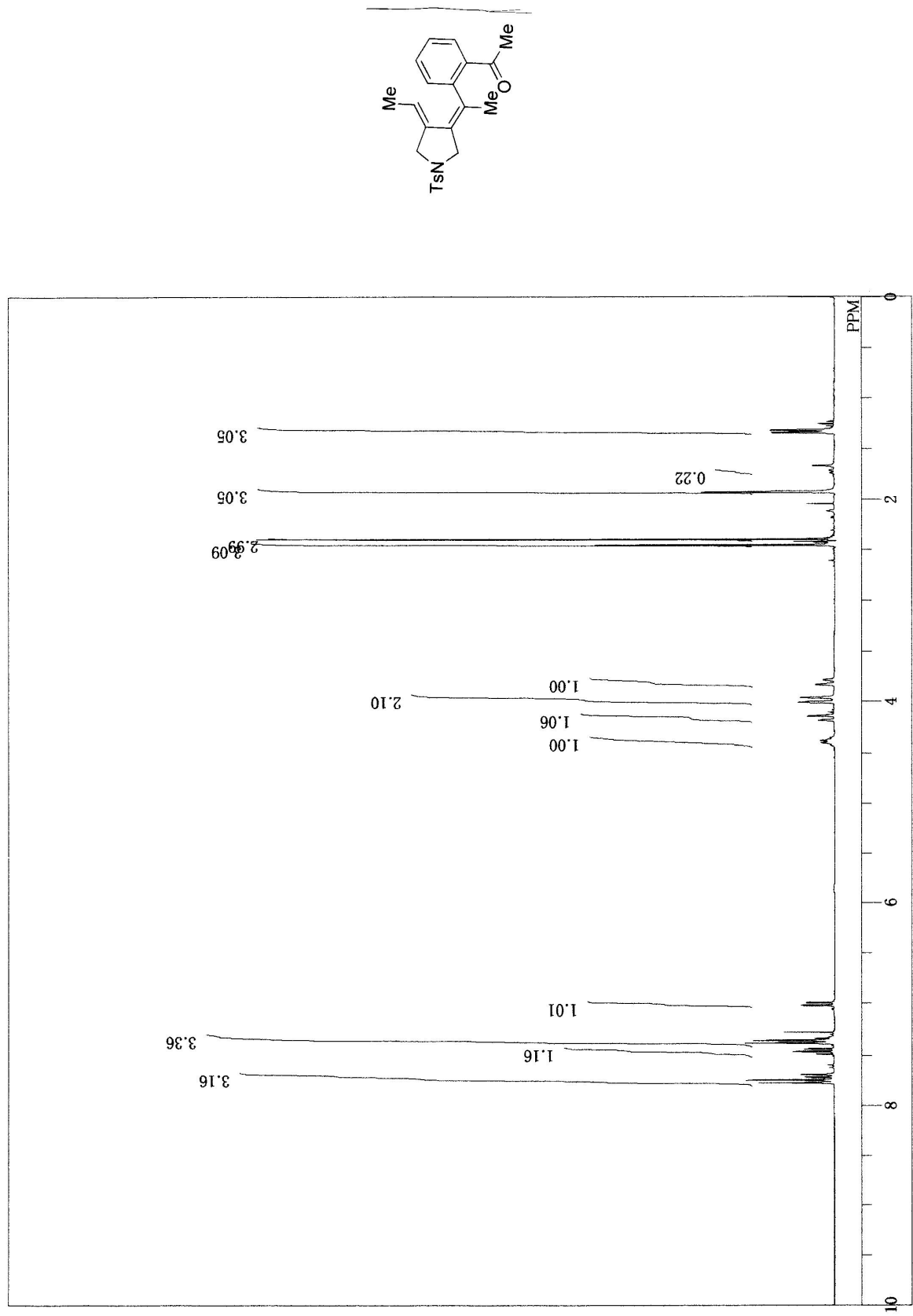
1-\{2-[1-(4-Ethylidenedihydrofuran-3-ylidene)ethyl]-4-methoxyphenyl\}ethanone $\quad(5 \mathrm{dl}$, $E: Z=18: 82$ )
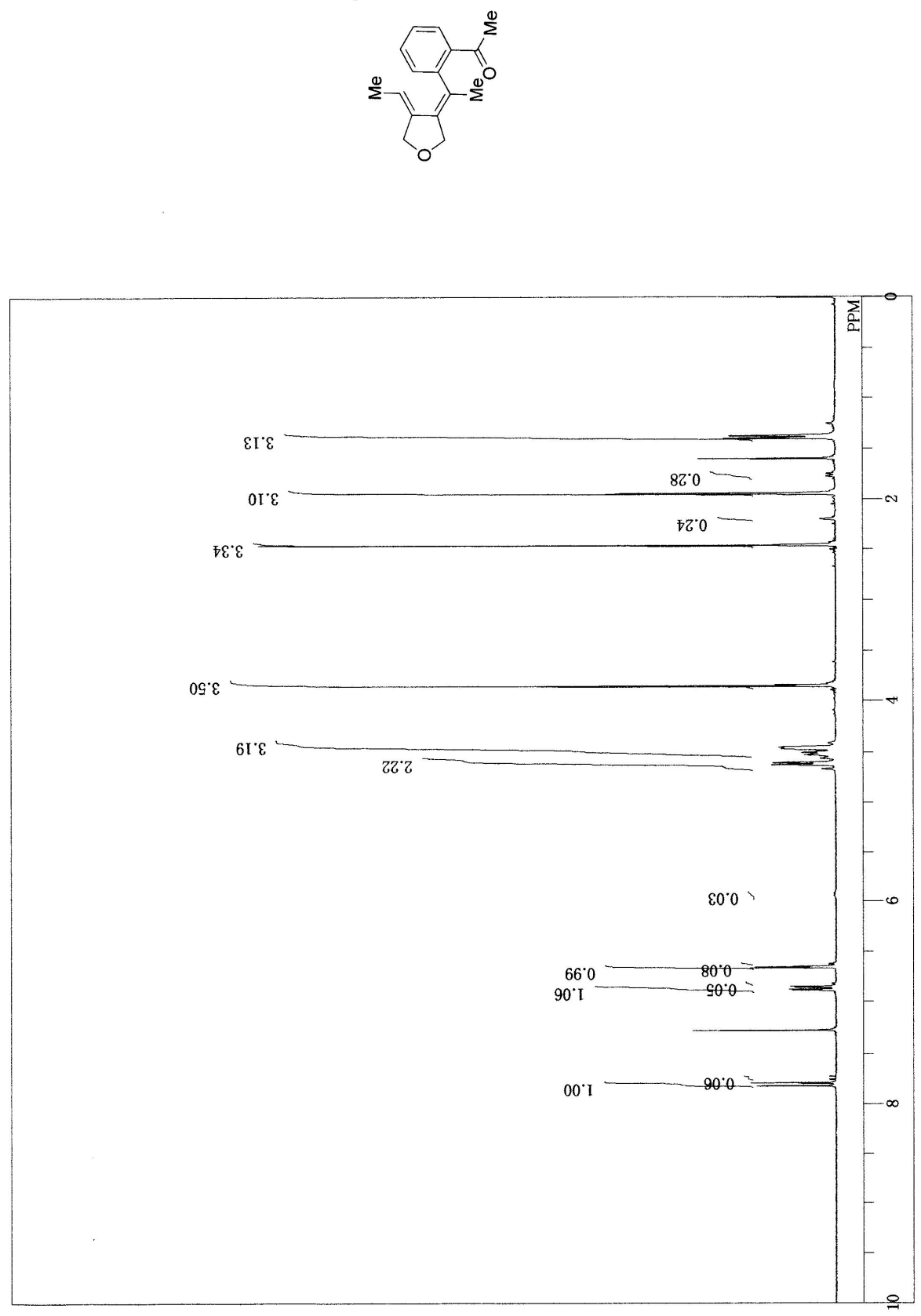
1-[2-\{1-(4-Ethylfuran-3-yl)ethyl\}-4-methoxyphenyl]ethanone (6)
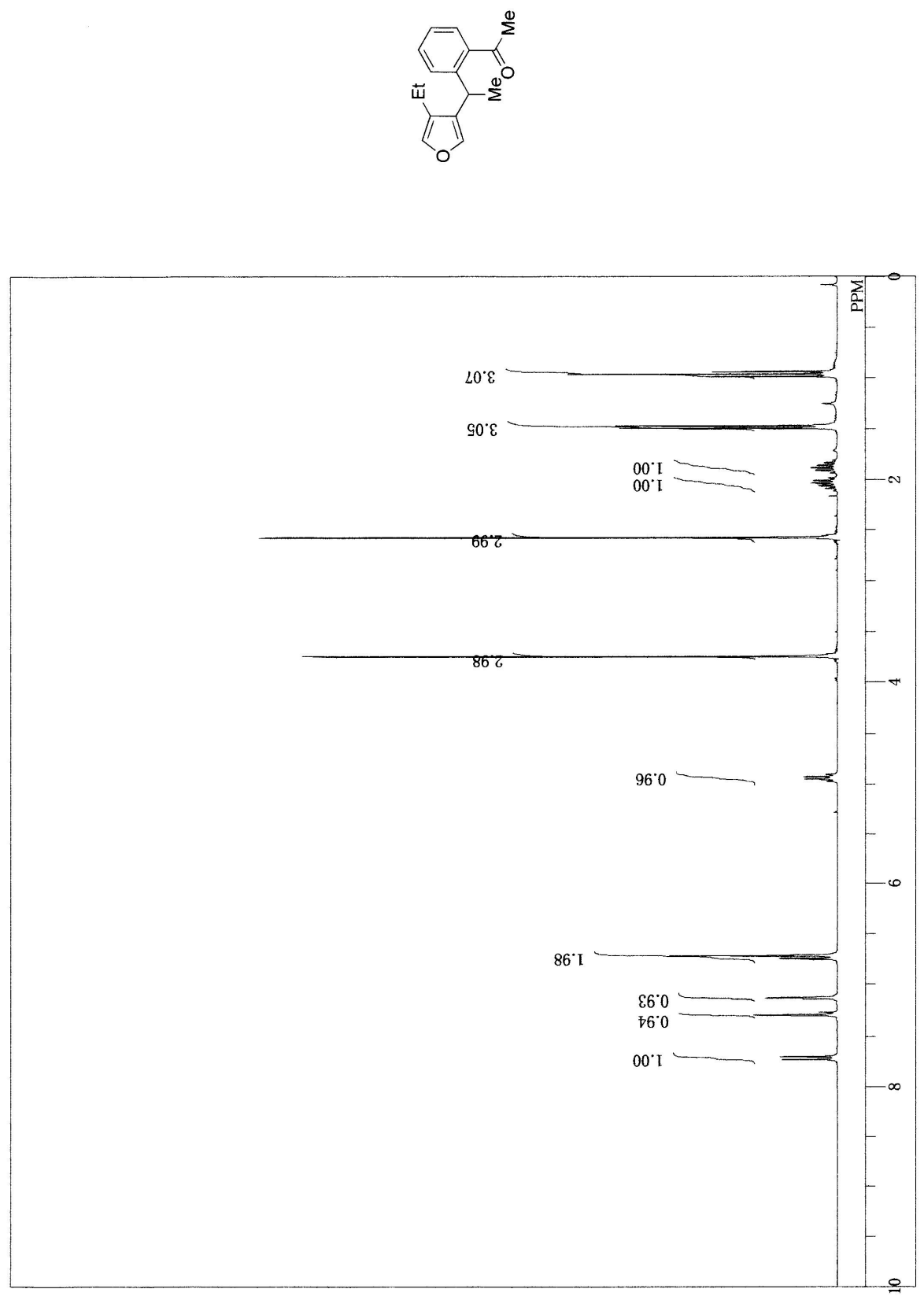
3-[1-(2-Acetyl-4-methoxyphenyl)ethylidene]-4-ethylidenecyclopentane-1,1dicarboxylic acid diethyl ester (5aq, $E: Z=6: 1$ )
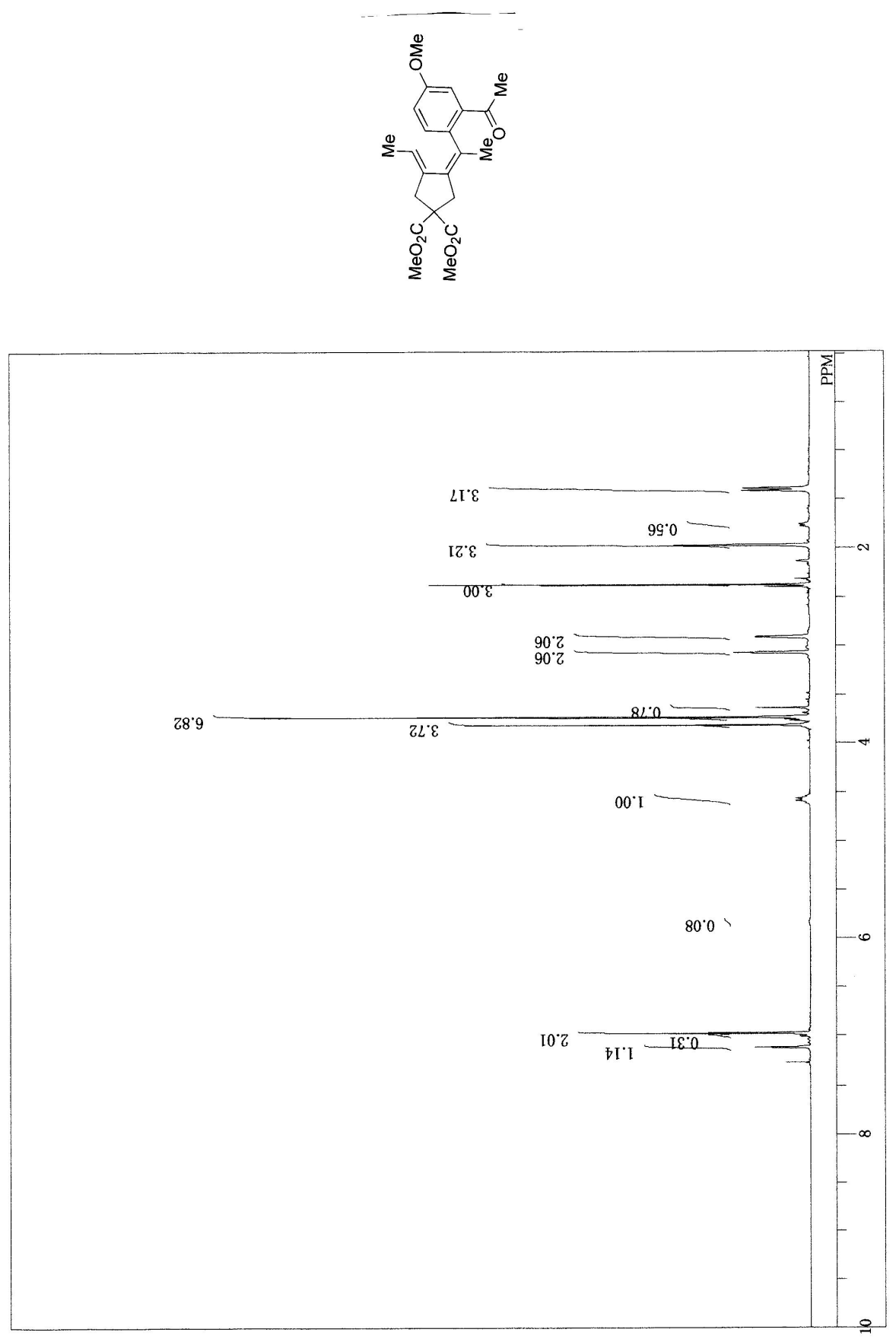
3-[1-(2-Acetyl-4,6-dimethoxyphenyl)ethylidene]-4-ethylidenecyclopentane-1,1dicarboxylic acid diethyl ester (5ar, $E: Z=>20: 1)$
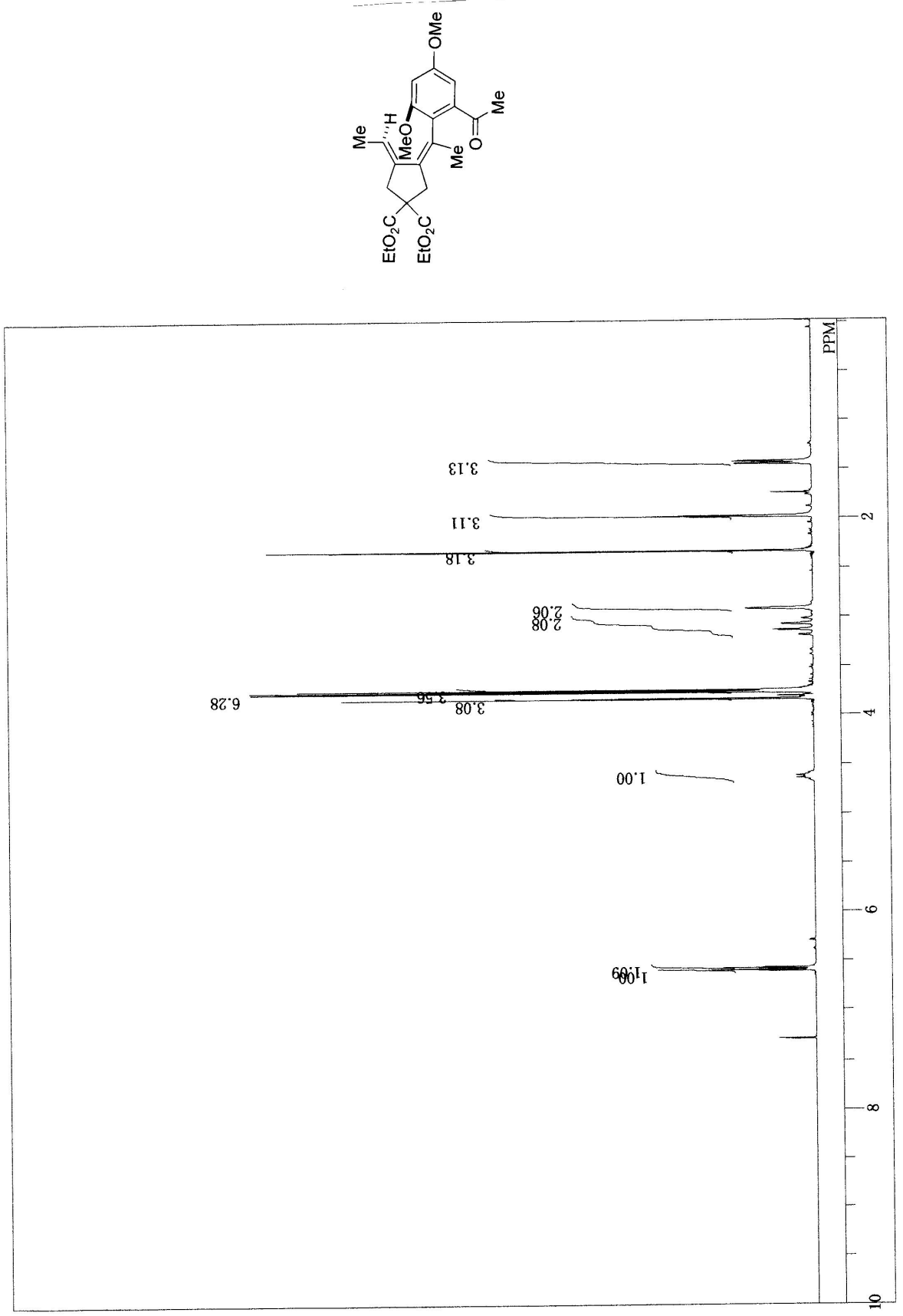
3-Acetyl-4-[2-(3,5-dimethoxyphenyl)-1-methylpropenyl]cyclopent-3-ene-1,1dicarboxylic acid dimethyl ester (3ar, $E: Z=2: 1$ )
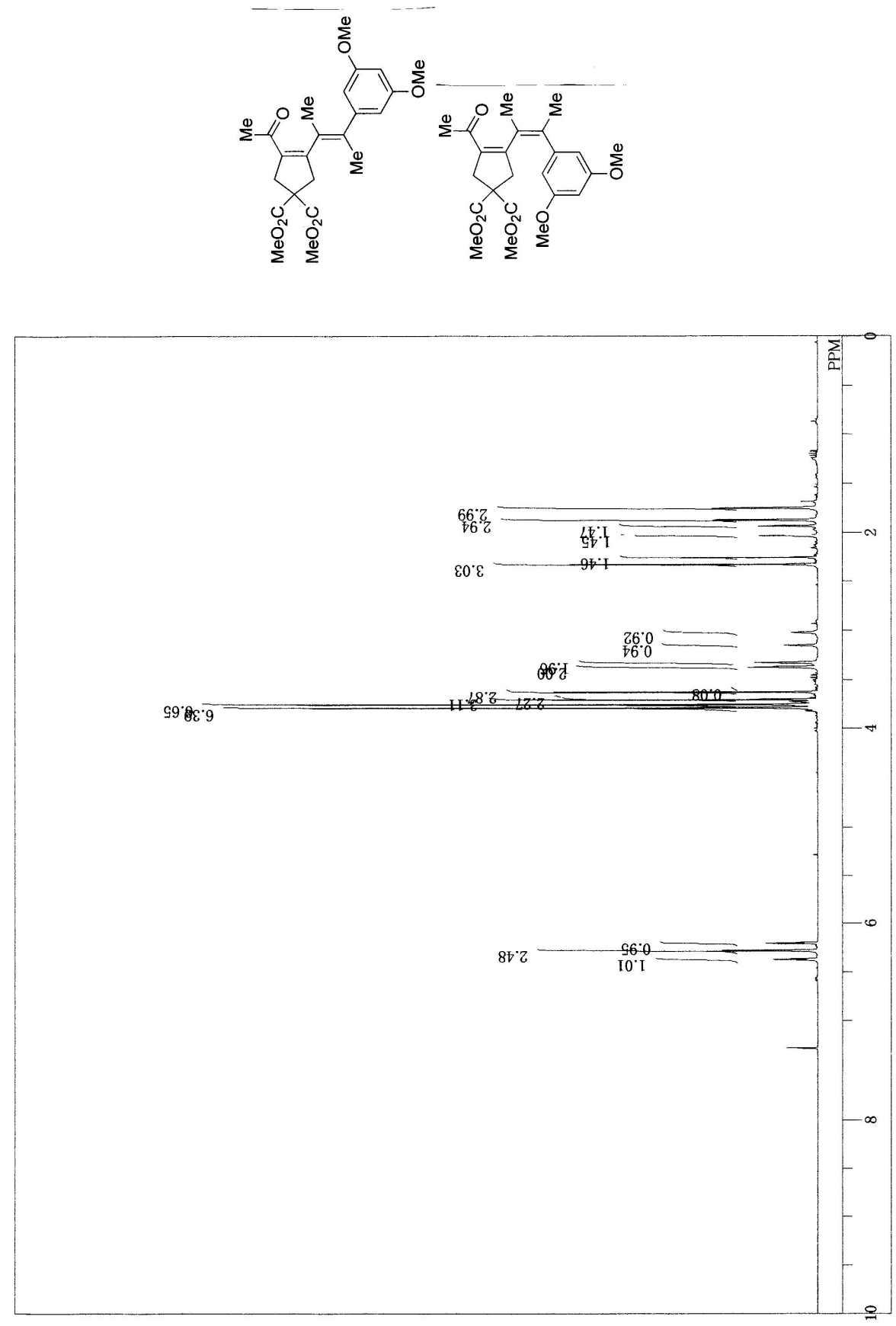